\title{
X-ray study of the double radio relic Abell 3376 with Suzaku
}

\author{
I. Urdampilleta ${ }^{1,2}$, H. Akamatsu ${ }^{1}$, F. Mernier ${ }^{1,3,4}$, J. S. Kaastra ${ }^{1,2}$, J. de Plaa ${ }^{1}$, T. Ohashi ${ }^{5}$, \\ Y. Ishisaki ${ }^{5}$, and H. Kawahara ${ }^{6,7}$
}

\author{
${ }^{1}$ SRON Netherlands Institute for Space Research, Sorbonnelaan 2, 3584 CA Utrecht, The Netherlands \\ e-mail: i.urdampilleta@sron.nl \\ 2 Leiden Observatory, Leiden University, PO Box 9513, 2300 RA Leiden, The Netherlands \\ 3 MTA-Eötvös University Lendület Hot Universe Research Group, Pázmány Péter sétány 1/A, Budapest 1117, Hungary \\ ${ }^{4}$ Institute of Physics, Eötvös University, Pázmány Péter sétány 1/A, Budapest 1117, Hungary \\ 5 Department of Physics, Tokyo Metropolitan University, 1-1 Minami-Osawa, Hachioji, Tokyo 192-0397, Japan \\ 6 Department of Earth and Planetary Science, The University of Tokyo, Tokyo 113-0033, Japan \\ 7 Research Center for the Early Universe, School of Science, The University of Tokyo, Tokyo 113-0033, Japan
}

Received 19 December 2017 / Accepted 20 June 2018

\begin{abstract}
We present an X-ray spectral analysis of the nearby double radio relic merging cluster Abell $3376(z=0.046)$, observed with the Suzaku XIS instrument. These deep ( $\sim 360 \mathrm{ks})$ observations cover the entire double relic region in the outskirts of the cluster. These diffuse radio structures are amongst the largest and arc-shaped relics observed in combination with large-scale X-ray shocks in a merging cluster. We confirm the presence of a stronger shock $\left(\mathcal{M}_{\mathrm{W}}=2.8 \pm 0.4\right)$ in the western direction at $r \sim 26^{\prime}$, derived from a temperature and surface brightness discontinuity across the radio relic. In the east, we detect a weaker shock $\left(\mathcal{M}_{\mathrm{E}}=1.5 \pm 0.1\right)$ at $r \sim 8^{\prime}$, possibly associated with the "notch" of the eastern relic, and a cold front at $r \sim 3^{\prime}$. Based on the shock speed calculated from the Mach numbers, we estimate that the dynamical age of the shock front is $\sim 0.6$ Gyr after core passage, indicating that Abell 3376 is still an evolving merging cluster and that the merger is taking place close to the plane of the sky. These results are consistent with simulations and optical and weak lensing studies from the literature.
\end{abstract}

Key words. X-rays: galaxies: clusters - shock waves

\section{Introduction}

Galaxy clusters form hierarchically by the accretion and merging of the surrounding galaxy groups and subclusters. During these energetic processes, the intracluster medium (ICM) becomes turbulent and produces cold fronts, the interface between the infalling cool dense gas core of the subcluster and the hot cluster atmosphere, and shock waves, which propagate into the intracluster medium of the subclusters (Markevitch \& Vikhlinin 2007). Part of the kinetic energy involved in the merger is converted into thermal energy by driving these large-scale shocks and turbulence, and the other part is transformed into nonthermal energy. Shocks are thought to (re)accelerate electrons from the thermal distribution up to relativistic energies by the firstorder Fermi diffusive shock acceleration mechanism (hereafter DSA, Bell 1987; Blandford \& Eichler 1987). The accelerated electrons, in the presence of a magnetic field, may produce radio relics via synchrotron radio emission (Ferrari et al. 2008, 2012; Brunetti \& Jones 2014). They are generally Mpc-scale sized, elongated and steep-spectrum radio structures (Brüggen et al. 2012; Brunetti \& Jones 2014), which appear to be associated with the shock fronts at the outskirts of merging clusters (Finoguenov et al. 2010; Ogrean \& Brüggen 2013).

Discoveries of shocks coinciding with radio relics are increasing (Markevitch et al. 2005; Finoguenov et al. 2010; Macario et al. 2011; Ogrean \& Brüggen 2013; Bourdin et al. 2013; Eckert et al. 2016b; Sarazin et al. 2016; Akamatsu \& Kawahara 2013; Akamatsu et al. 2015, 2017). However, not all merging clusters present the same spatial distribution of the X-ray and radio components (Ogrean et al. 2014; Shimwell et al. 2016). The study of the radial distribution of these thermal and nonthermal components allows us to estimate the dynamical stage of the cluster as well as to understand how the shock propagates and heats the ICM. It can also determine the physical association between radio relics and shocks. The lack of connection between radio relics and shocks in several merging clusters, together with the low efficiency of DSA for $\mathcal{M} \sim 2-3$ of these shocks, seems to suggest that in some cases the DSA assumption is not enough for the electron acceleration from the thermal pool (Vink \& Yamazaki 2014; Vazza \& Brüggen 2014; Skillman et al. 2013; Pinzke et al. 2013). For this reason, alternative mechanisms have recently been proposed, such as for example, the re-acceleration of pre-existing cosmic ray electrons (Markevitch et al. 2005; Kang et al. 2012; Fujita et al. 2015, 2016; Kang 2017) and shock drift acceleration (Guo et al. 2014a,b, 2017). However, it is not clear what type of mechanism is involved and whether all relics need additional mechanisms or not. Some merging clusters present different results, as for example "El Gordo" (Botteon et al. 2016b) is consistent with the DSA mechanism. However, A3667 South (Storm et al. 2018), CIZA J2242.8+5301 (Hoang et al. 2017) and A3411-3412 (van Weeren et al. 2017) challenge the direct shock acceleration of electrons from the thermal pool. Therefore, it is not possible to provide a definitive explanation for these discrepancies.

In this paper, we analyze the spatial distribution of thermal and nonthermal components in Abell 3376, (hereafter A3376), based on Suzaku observations (Mitsuda et al. 2007). We have also used XMM-Newton and Chandra observations to support 

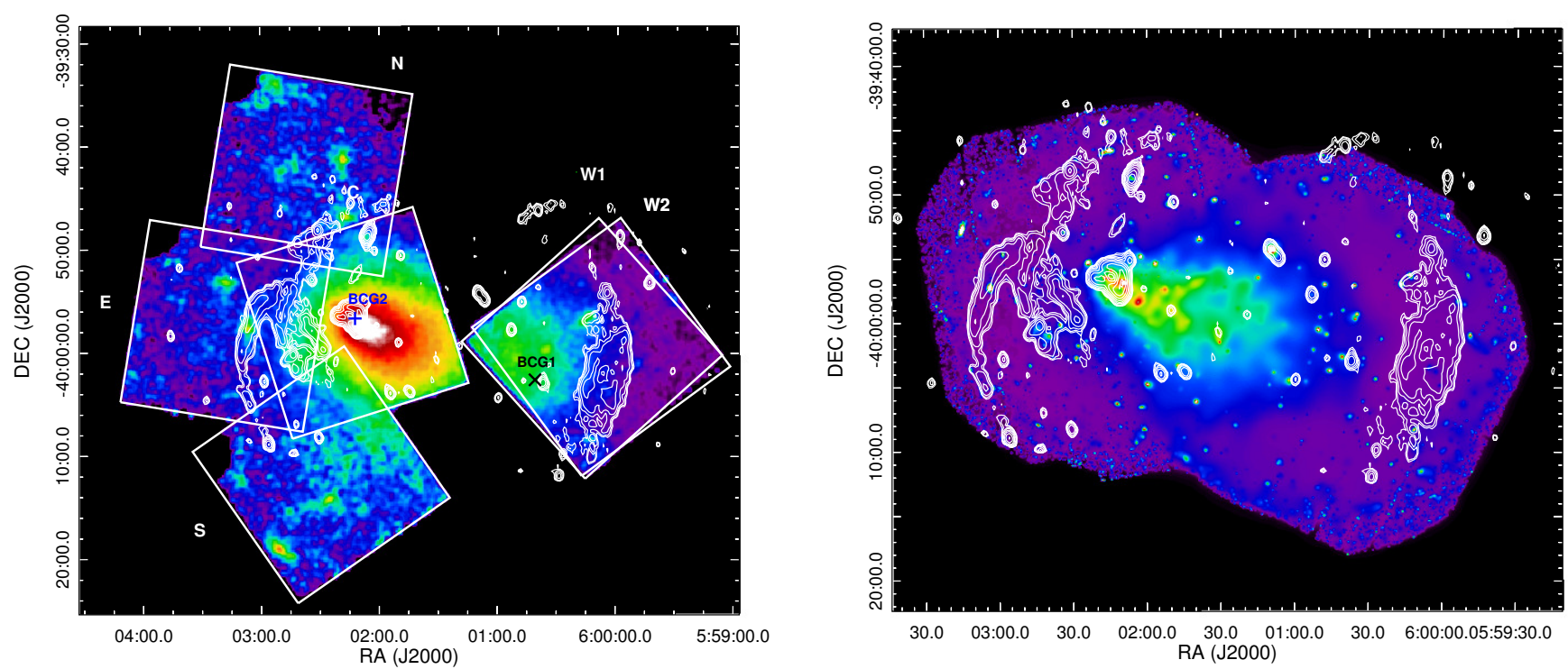

Fig. 1. Left: Suzaku smoothed image in the $0.5-10 \mathrm{keV}$ band of A3376 (pixel $=8^{\prime \prime}$ and Gausssian 2-D smooth $\sigma=16^{\prime \prime}$ ). The white boxes represent the Suzaku XIS FOVs for the C, W1, W2, N, E and S observations listed in Table 1. BCG1 is shown as a black cross and BCG2 as a blue cross. Right: XMM-Newton image in the $0.5-10 \mathrm{keV}$ band of A3376. The white contours correspond to VLA radio observations kindly provided by Dr. R. Kale.

Table 1. Observations and exposure times.

\begin{tabular}{|c|c|c|c|c|c|c|}
\hline Observatory & Name & Sequence ID & $\begin{array}{c}\text { Position (J2000) } \\
\quad(\text { RA, Dec) }\end{array}$ & $\begin{array}{l}\text { Observation } \\
\text { starting date }\end{array}$ & $\begin{array}{c}\text { Exp. }^{a} \\
(\mathrm{ks})\end{array}$ & $\begin{array}{l}\text { Exp. }^{b} \\
(\mathrm{ks})\end{array}$ \\
\hline \multirow[t]{7}{*}{ Suzaku } & $\mathrm{C}$ & 100043010 & $(90.56,-39.94)$ & $2005-10-06$ & 110.4 & 76.8 \\
\hline & W1 & 800011010 & $(90.05,-39.98)$ & $2005-11-07$ & 119.4 & 99.4 \\
\hline & W2 & 800011020 & $(90.04,-39.98)$ & $2006-10-26$ & 56.8 & 44.1 \\
\hline & $\mathbf{N}^{c}$ & 808029010 & $(90.64,-39.70)$ & 2013-09-18 & 51.4 & 33.9 \\
\hline & $\mathrm{E}^{c}$ & 808028010 & $(90.81,-39.95)$ & 2013-09-14 & 71.7 & 51.4 \\
\hline & $\mathrm{S}^{c}$ & 808030010 & $(90.61,-40.18)$ & $2013-10-15$ & 61.3 & 44.5 \\
\hline & Q0551-3637 & 703036020 & $(88.19,-36.63)$ & 2008-05-14 & 18.8 & 15.3 \\
\hline \multirow[t]{2}{*}{ XMM-Newton } & XMM-E & 0151900101 & $(90.54,-39.96)$ & 2003-04-01 & 47.2 & 33.1 \\
\hline & XMM-W & 0504140101 & $(90.21,-40.03)$ & 2007-08-24 & 46.1 & 40.8 \\
\hline Chandra & Chandra & 3202 & $(90.54,-39.96)$ & $2002-03-16$ & 44.3 & - \\
\hline
\end{tabular}

Notes. ${ }^{(a)}$ Suzaku data screening without COR2, XMM-Newton and Chandra total exposure time. ${ }^{(b)}$ Suzaku data screening with COR2 > 8 GV; XMM-Newton data screening. ${ }^{(c)}$ Additional processing for XIS1.

and confirm the results obtained with Suzaku. A3376 is a nearby $(z=0.046$, Struble \& Rood 1999), bright and moderately massive $\left(M_{200} \sim 4-5 \times 10^{14} M_{\odot}\right.$, Durret et al. 2013; MonteiroOliveira et al. 2017) merging galaxy cluster. This merging system has two giant $(\sim \mathrm{Mpc})$ arc-shaped radio relics in the cluster outskirts, discovered by Bagchi et al. (2006). The radio observations (Bagchi et al. 2006; Kale et al. 2012; George et al. 2015), see Fig. 1, reveal complex radio structures at the western and eastern directions. In the west, A3376 shows a lowly polarized wide $(\sim 300 \mathrm{kpc})$ relic with a non-aligned magnetic field (Kale et al. 2012). In the east, the relic is divided in three parts: the northern faint relic with steep spectral index; an elongated and polarized bright relic; and an additional "notch" with a $\sim 500 \mathrm{kpc}$ extension toward the center $(\alpha \sim-1.5)$ (Kale et al. 2012; Paul et al. 2011). Radio spectral index studies, assuming diffusive shock acceleration, with an estimated $\mathcal{M} \sim 2-3$, which is consistent with the previous X-ray study by Akamatsu et al. (2012) of the western relic. A3376 includes two brightest cluster galaxies (BCG), coincident with two overdensities in projected galaxy distribution. BCG1 (ESO $307-13, \mathrm{RA}=6^{\mathrm{h}} 00^{\mathrm{m}} 41 \mathrm{~s} .10, \mathrm{Dec}=-40^{\circ} 02^{\prime} 40^{\prime} \cdot 00$ ) belongs to the west subcluster, while BCG2 (2MASX J06020973-3956597, $\mathrm{RA}=6^{\mathrm{h}} 02^{\mathrm{m}} 09$ s 70, Dec $=-39^{\circ} 57^{\prime} 05^{\prime \prime}$.00) is located close to the $\mathrm{X}$-ray peak emission at the east subcluster. The N-body hydrodynamical simulations of Machado \& Lima Neto (2013) reproduce a bimodal merger system, with a mass ratio of 3-6:1, formed by one compact and dense subcluster, which crossed at high velocity and disrupted the core of the second massive subcluster $\sim 0.5-0.6$ Gyr ago. This merger scenario was later corroborated by the optical analysis of Durret et al. (2013) and the weak lensing study of Monteiro-Oliveira et al. 2017.

For this study, we used the values of protosolar abundances $\left(Z_{\odot}\right)$ reported by Lodders et al. (2009). The abundance of all metals are coupled to Fe. We used a Galactic absorption column of $N_{\mathrm{H} \text {,total }}=5.6 \times 10^{20} \mathrm{~cm}^{-2}$ (Willingale et al. 2013) for all the fits. We assumed cosmological parameters $H_{0}=70 \mathrm{~km} \mathrm{~s}^{-1} \mathrm{Mpc}^{-1}$, $\Omega_{\mathrm{M}}=0.27$ and $\Omega_{\Lambda}=0.73$, respectively, which give $54 \mathrm{kpc}$ per 


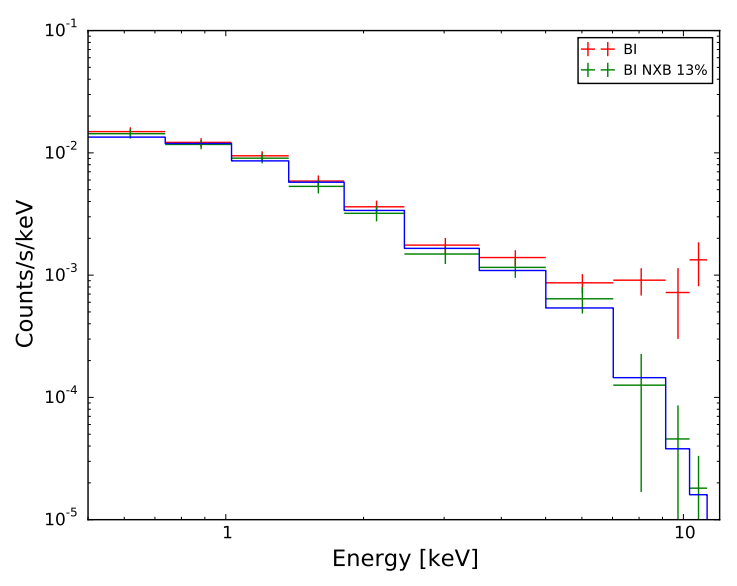

Fig. 2. Correction of the XIS1 excess in the outer region of the E observation of A3376. The best-fit ICM spectrum is shown as a blue line. The Suzaku XIS BI spectra are shown without (red) and with (green) a $13 \%$ increase in the NXB level, respectively.

$1 \operatorname{arcmin}$ at $z=0.046$. The virial radius is adopted as $r_{200}$, the radius where the mean interior density is 200 times the critical density at the redshift of the source. For our cosmology and redshift we used the approximation of Henry et al. (2009):

$r_{200}=2.77 h_{70}^{-1}(\langle k T\rangle / 10 \mathrm{keV})^{1 / 2} / E(z) \mathrm{Mpc}$,

where $E(z)=\left(\Omega_{\mathrm{M}}(1+z)^{3}+1-\Omega_{\mathrm{M}}\right)^{1 / 2}, r_{200}$ is $1.76 \mathrm{Mpc}\left(\sim 32.6^{\prime}\right)$ with $\langle k T\rangle=4.2 \mathrm{keV}$ as calculated by Reiprich et al. (2013). All errors are given at $1 \sigma(68 \%)$ confidence level unless otherwise stated and all the spectral analysis made use of the modified Cash statistics (Cash 1979; Kaastra 2017).

\section{Observations and data reduction}

Table 1 and Fig. 1 summarize the observations used for the present analysis. As shown in Fig. 1 six different Suzaku pointings have been used together with two additional XMM-Newton observations. Three of the Suzaku pointings are located in the central and western region (hereafter named as C, W1 and W2), which were obtained in 2005 and 2006, respectively. The other three observations (2013) cover the north (N), east (E), and south (S) of the outer eastern region. The combined observations cover the complete relic structures of A3376 as the western relic (W1 and W2), center (C) and eastern relic (N, E, and S). The total effective exposure time of the Suzaku data after screening and filtering of the cosmic-ray cut-off rigidity (COR2, Tawa et al. 2008) is $2360 \mathrm{ks}$.

All observations have been performed with the normal $3 \times 3$ or $5 \times 5$ clocking mode of the X-ray imaging spectrometer (hereafter XIS, Koyama et al. 2007). This instrument had four active detectors: XIS0, XIS2 and XIS3 (front-side illuminated, FI) and XIS1 (back-side illuminated, BI). On November 9, 2006 the XIS2 detector suffered a micrometeorite strike ${ }^{1}$ and was no longer operative. The XIS0 detector had a similar accident in 2010 and a part of the segment A was damaged ${ }^{2}$. In the same year the amount of charge injection for XIS1 was increased from 2 to $6 \mathrm{keV}$, which leads to an increase in the non-X-ray

\footnotetext{
1 http://www.astro.isas.ac.jp/suzaku/doc/suzakumemo/ suzakumemo-2007-08.pdf

2 http://wWw.astro.isas.ac.jp/suzaku/doc/suzakumemo/ suzakumemo-2010-07v4.pdf
}

background (NXB) level of XIS1. For that reason an additional screening has been applied to minimize the NXB level following the process described in the Suzaku Data Reduction Guide ${ }^{3}$.

We used HEAsoft version 6.20 and CALDB 2016-01-28 for all Suzaku analysis presented here. We have applied standard data screening with the elevation angle $>10^{\circ}$ above the Earth and cut-off rigidity (COR2) $>8 \mathrm{GV}$ to increase the signal to noise. The energy range of $1.7-1.9 \mathrm{keV}$ was ignored in the spectral fitting owing to the residual uncertainties present in the Si-K edge.

For the point sources identification and exclusion, we used XMM-Newton observations (ID: 0151900101 and 0504140101, see Table 1). We applied the data reduction software SAS v14.0.0 for the XMM-Newton EPIC instrument with the task named edetect_chain. We used visual inspection for the point sources identification beyond the XMM-Newton observations in the east. We derived the surface brightness (SB) profiles from the XMM-Newton and Chandra (ID: 3202, see Table 1) observations. We used CIAO 4.8 with CALDB 4.7.6 for the data reduction of Chandra observations. Moreover, the Suzaku offset observation of Q0551-3637, located at $\sim 4^{\circ}$ distance from A3376, was analyzed to estimate the sky background components as described in the following section.

\section{Spectral analysis and results}

\subsection{Spectral analysis approach}

In our spectral analysis of A3376, we have assumed that the observed spectra include the following components: optically thin thermal plasma emission from the ICM, the cosmic X-ray background (CXB), the local hot bubble (LHB), the Milky Way halo (MWH) and non X-ray background (NXB). We first generated the non X-ray background spectra using xisnxbgen (Tawa et al. 2008). Secondly, we identified the point sources present in our data using the two observations of XMM-Newton (see Table 1) and extracted them with a radius of $1-3^{\prime}$ from the Suzaku observations. We have estimated the sky background emission consisting of CXB, LHB, and MWH from the Q05513637 observation at $3.8^{\circ}$ from A3376 (see Sect. 3.2). We assumed that the sky background component is uniformly distributed along the A3376 extension. We generated the redistribution matrix file (RMF) using xisrmfgen and the ancillary response file (ARF) with xisimarfgen (Ishisaki et al. 2007) considering a uniform input image $\left(r=20^{\prime}\right)$.

A proper estimation of the background components is crucial, specially in the outskirts of galaxy clusters, where usually the X-ray emission of the ICM is low and the spectrum can be background dominated. The $\mathrm{X}$-ray shock fronts and radio relics are usually located at these outer regions.

We performed a temperature deprojection assuming spherical symmetry to check the projection effect on the temperature profiles. We applied the method described in Blanton et al. (2003) for the post-shock regions, which are affected by projection of the emission of the outer and cooler regions. The results of the deprojection fits are consistent within $1 \sigma$ uncertainties with the projected temperatures. For this reason, we used the projected fits in this work.

In our spectral analysis, we used $\operatorname{SPEX}^{4}$ (Kaastra et al. 1996) version 3.03.00 with SPEXACT version 2.07.00. We carried out

\footnotetext{
3 https://heasarc.gsfc.nasa.gov/docs/suzaku/analysis/ abc/node8.html

4 https://wWW. sron.nl/astrophysics-spex
} 


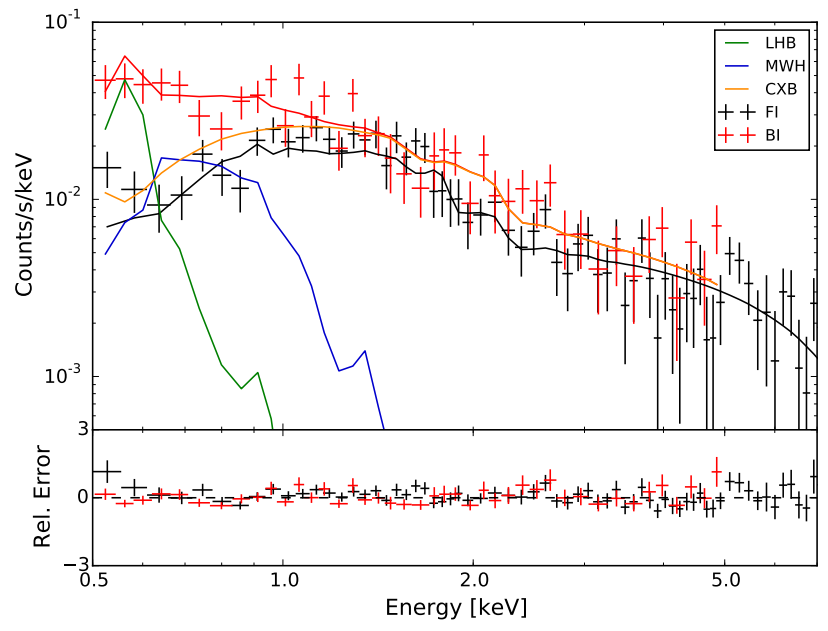

Fig. 3. Q0551-3637 spectrum used for the sky background estimation The Suzaku XIS FI, BI, the LHB, MWH and CXB components are shown in black, red, green, blue, and orange, respectively. The LHB, $\mathrm{MWH}$, and CXB have been represented relative to the BI spectrum.

Table 2. Background components derived from the Q0551-3637 observation.

\begin{tabular}{cccc}
\hline \hline & LHB & MWH & CXB \\
\hline Norm $^{a}$ & $108 \pm 21$ & $2.9 \pm 0.5$ & $4.9 \pm 0.4$ \\
$k T(\mathrm{keV})$ & 0.08 (fixed) & 0.27 (fixed) & - \\
$\Gamma$ & - & - & 1.41 (fixed) \\
\hline C-stat/d.o.f. & & $130 / 103$ & \\
\hline
\end{tabular}

Notes. ${ }^{(a)}$ For LHB and MWH norm in units of $10^{71} \mathrm{~m}^{-3}$ scaled by $400 \pi$. For CXB norm in units of $10^{51} \mathrm{phs}^{-1} \mathrm{keV}^{-1}$.

the spectral fitting in different annular regions as detailed in the sections below. The spectra of the XIS FI and BI detectors were fitted simultaneously and binned using the method of optimal binning (Kaastra \& Bleeker 2016). For all the spectral fits, the NXB component is subtracted using the trafo tool in SPEX. The free parameters considered in this study are the temperature $k T$, the normalization Norm and the metal abundance $\mathrm{Z}$ for the inner regions $r \leq 9^{\prime}$ of the ICM component. For the outer regions $\left(r>10^{\prime}\right)$ we fixed the abundance to $0.3 Z_{\odot}$ as explained by Fujita et al. (2008) and Urban et al. (2017).

\subsection{Estimation of the background spectra}

The NXB component was obtained as the weighted sum of the XIS Night Earth observations and it was compiled for the same detector area and COR2 condition (COR2 $>8 \mathrm{GV})$ during 150 days before and after the observation date. In this way the long-term variation of the XIS detector background due to radiation damage can be constrained. The systematic errors corresponding to the NXB intensity are considered to be $\pm 3 \%$ (Tawa et al. 2008). For the most recent observations (E, N, and $\mathrm{S}$ ), where a new spectral analysis approach was proposed for $\mathrm{XIS} 1^{5}$ because of the charge injection increase to $6 \mathrm{keV}$, we detected an excess of source counts in the high energy band $(>10 \mathrm{keV})$. We managed to compensate for this excess by

\footnotetext{
5 https://heasarc.gsfc.nasa.gov/docs/suzaku/analysis/ $\mathrm{abc} /$ node $8 \cdot \mathrm{html}$
}

increasing the NXB level by $13 \%$. In this way the source level (ICM) follows the spectral shape of the CXB at high energies (see Fig. 2).

As mentioned in Sect.2, the point source identification in the Suzaku FOV was realized using two XMM-Newton observations. The SAS task named edetect_chain was applied in four different bands $(0.3-2.0 \mathrm{keV}, 2.0-4.5 \mathrm{keV}, 4.5-7.5 \mathrm{keV}$, and 7.5-12 keV) using EPIC pn and MOS data. We used a simulated maximum likelihood value of ten. After this, we combined the detections in these four bands and estimated the flux of each source in a circle with a radius of $0.6^{\prime}$. We established the minimum of flux detection of the extracted sources as $S_{\mathrm{c}}=$ $10^{-17} \mathrm{~W} \mathrm{~m} \mathrm{~m}^{-2}$ in the energy band $2-10 \mathrm{keV}$. Although this limit is lower than the level reported by Kushino et al. (2002) $S_{\mathrm{c}}=2 \times 10^{-16} \mathrm{~W} \mathrm{~m}^{-2}$, we obtained an acceptable $\log N-\log S$ distribution contained within the Kushino et al. (2002) limits (see their Fig. 20). We excluded in the Suzaku observations the identified point sources with a radius of 1 arcmin in order to account for the point spread function (PSF) of XIS (Serlemitsos et al. 2007). As a result, our CXB intensity after the point sources extraction for the $2-10 \mathrm{keV}$ band was estimated as $5.98 \times 10^{-11} \mathrm{~W} \mathrm{~m}^{-2} \mathrm{sr}^{-1}$. This value is within $\pm 10 \%$ agreement with Cappelluti et al. (2017), Akamatsu et al. (2012) and Kushino et al. (2002).

As a sanity check, we compared the sky background level of ROSAT observations for the outermost region of A3376 $\left(r=30^{\prime}-60^{\prime}\right)$ and the offset pointing Q0551-3637 $\left(r=3^{\prime}-20^{\prime}\right)$ using the HEASARC X-ray Background Tool $^{6}$ in the band R45 (0.4-1.2 keV). This band contains most of the emission of the sky background. The R45 intensities in the units of $10^{-6}$ counts s$^{-1}$ arcmin $^{-2}$ are $125.3 \pm 5.0$ for the A3376 outer ring and $114.4 \pm 18.5$ for Q0551-3637. Both values are in good agreement.

We used the same $S_{\mathrm{c}}=10^{-17} \mathrm{~W} \mathrm{~m}^{-2}$ for extracting the point sources in the offset Suzaku observation of Q0551-3637. We also excluded a circle of $3^{\prime}$ centered around the quasar with the same name, which is the brightest source of this region. Thereafter, we fitted the resultant spectra using $N_{\mathrm{H}}=3.2 \times 10^{20} \mathrm{~cm}^{-2}$ (Willingale et al. 2013), considering the emission of three sky background components at redshift zero and metal abundance of one. For the FI and BI detectors we used the energy ranges of $0.5-7.0 \mathrm{keV}$ and $0.5-5.0 \mathrm{keV}$, respectively. The two Galactic components are modeled with: LHB (fixed $k T=$ $0.08 \mathrm{keV}$ ), unabsorbed cie model (collisional ionization equilibrium in SPEX) and MWH (fixed $k T=0.27 \mathrm{keV}$ ), absorbed cie. The third component is the CXB modeled as an absorbed powerlaw with fixed $\Gamma=1.41$. The complete sky background model is:

$\operatorname{cie}_{\mathrm{LHB}}+a b s *\left(\right.$ cie $_{\mathrm{MWH}}+$ powerlaw $\left._{\mathrm{CXB}}\right)$.

Best-fit parameters are listed in Table 2, and the sky background components are shown in Fig. 3. For the calculation of the systematic uncertainties of the CXB fluctuation due to unresolved point sources $\left(\sigma / I_{\mathrm{CXB}}\right)$ we have used Eq. (3) proposed by Hoshino et al. (2010) in each spatial region of Fig. 4 and Fig. 7 as

$\frac{\sigma}{I_{\mathrm{CXB}}}=\frac{\sigma_{\text {Ginga }}}{I_{\mathrm{CXB}}}\left(\frac{\Omega_{\mathrm{e}}}{\Omega_{\mathrm{e}, \text { Ginga }}}\right)^{-0.5}\left(\frac{S_{\mathrm{c}}}{S_{\mathrm{c}, \text { Ginga }}}\right)^{0.25}$,

where $\left(\sigma_{\text {Ginga }} / I_{\mathrm{CXB}}\right)=5, \Omega_{\mathrm{e}, \text { Ginga }}=1.2 \mathrm{deg}^{2}, S_{\mathrm{c}, \text { Ginga }}=6 \times$ $10^{-15} \mathrm{~W} \mathrm{~m}^{-2}, S_{\mathrm{c}}=10^{-17} \mathrm{~W} \mathrm{~m}^{-2}$ and $\Omega_{\mathrm{e}}$ is the effective solid

6 https://heasarc.gsfc.nasa.gov/cgi-bin/Tools/xraybg/ xraybg.pl 
Table 3. Best-fit parameters for the central and western regions shown in Fig. 4.

\begin{tabular}{cccccc}
\hline \hline & Radius $\left(^{\prime}\right)$ & $k T(\mathrm{keV})$ & Norm $\left(10^{73} \mathrm{~m}^{-3}\right)$ & $Z\left(Z_{\odot}\right)$ & C-stat/d.o.f. \\
\hline \multirow{6}{*}{ Center } & $1.0 \pm 1.0$ & $4.27 \pm 0.07$ & $7.92 \pm 0.11$ & $0.53 \pm 0.04$ & $803 / 793$ \\
& $3.0 \pm 1.0$ & $4.32 \pm 0.11$ & $7.32 \pm 0.16$ & $0.51 \pm 0.06$ & $590 / 598$ \\
& $5.0 \pm 1.0$ & $4.04 \pm 0.09$ & $6.32 \pm 0.15$ & $0.41 \pm 0.05$ & $634 / 606$ \\
& $7.5 \pm 1.5$ & $4.05 \pm 0.08$ & $5.52 \pm 0.09$ & $0.39 \pm 0.04$ & $606 / 583$ \\
\hline \multirow{6}{*}{ West } & $14.0 \pm 1.0$ & $4.29 \pm 0.18$ & $2.68 \pm 0.08$ & 0.3 (fixed) & $327 / 337$ \\
& $16.0 \pm 1.0$ & $4.34 \pm 0.18$ & $1.90 \pm 0.06$ & 0.3 (fixed) & $415 / 415$ \\
& $18.0 \pm 1.0$ & $4.34 \pm 0.19$ & $1.41 \pm 0.04$ & 0.3 (fixed) & $451 / 448$ \\
& $20.0 \pm 1.0$ & $5.04 \pm 0.23$ & $0.90 \pm 0.02$ & 0.3 (fixed) & $690 / 656$ \\
& $22.5 \pm 1.5$ & $4.52 \pm 0.24$ & $0.42 \pm 0.01$ & 0.3 (fixed) & $796 / 752$ \\
& $25.5 \pm 1.5$ & $2.85 \pm 0.34$ & $0.15 \pm 0.01$ & 0.3 (fixed) & $670 / 576$ \\
& $29.0 \pm 2.0$ & $1.44 \pm 0.27$ & $0.03 \pm 0.01$ & 0.3 (fixed) & $329 / 244$ \\
\hline
\end{tabular}

Table 4. Best-fit parameters for the pre- and post-shock regions at the western relic shown in Fig. 6.

\begin{tabular}{cccc}
\hline \hline & $k T(\mathrm{keV})$ & Norm $\left(10^{71} \mathrm{~m}^{-3}\right)$ & C-stat/d.o.f. \\
\hline Post & $4.22 \pm 0.26$ & $27.7 \pm 1.0$ & $680 / 654$ \\
Pre & $1.27 \pm 0.29$ & $2.3 \pm 0.8$ & $638 / 516$ \\
\hline
\end{tabular}

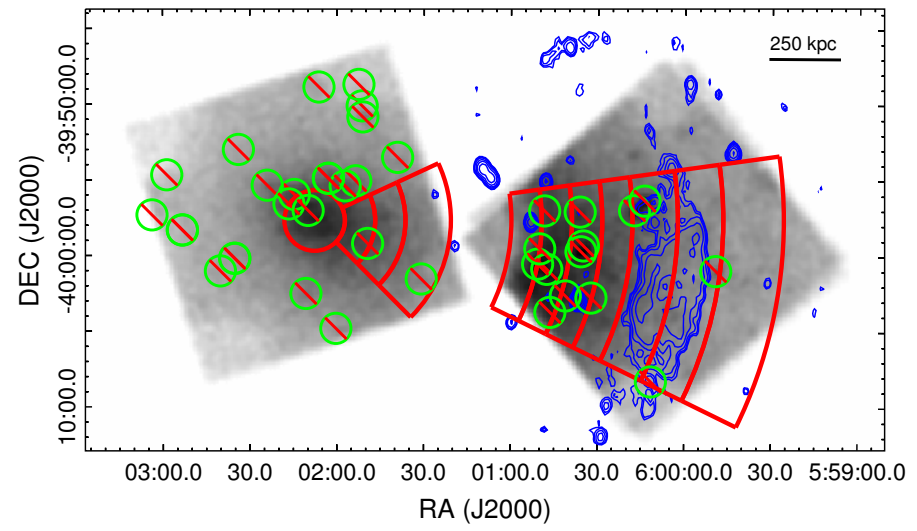

Fig. 4. A3376 central and western observations in the band $0.5-10 \mathrm{keV}$. The blue contours represent VLA radio observations. The green circles represent the extracted points sources with $r=1^{\prime}$. The red annular regions are used for the spectral analysis detailed in Table 3.

angle of the detector. This way, we have included the effect of systematic uncertainties related to the NXB intensity $( \pm 3 \%$, Tawa et al. 2008) and the CXB fluctuation, which varies between 8 and $27 \%$. The contributions of these systematic uncertainties are shown in Figs. 5 and 8.

\subsection{Spectral analysis along the western region}

For the spectral analysis of the western region, we have analyzed several annular regions centered on the X-ray emission peak centroid ( $\left.\mathrm{RA}=6^{\mathrm{h}} 02^{\mathrm{m}} 07 \mathrm{~s} .66, \mathrm{Dec}=-39^{\circ} 57^{\prime} 42^{\prime \prime} \cdot 74\right)$ once the point sources (see green circles in Fig. 4) have been excluded. The regions are a circle in the center with $r=2^{\prime}$ and annuli between $2^{\prime}-4^{\prime}, 4^{\prime}-6^{\prime}, 6^{\prime}-9^{\prime}, 13^{\prime}-15^{\prime}, 15^{\prime}-17^{\prime}, 17^{\prime}-19^{\prime}, 19^{\prime}-21^{\prime}$, $21^{\prime}-24^{\prime}, 24^{\prime}-27^{\prime}$, and $27^{\prime}-31^{\prime}$. For all of them, the FI (used energy range $0.5-10 \mathrm{keV})$ and $\mathrm{BI}(0.5-7.0 \mathrm{keV})$ spectra have

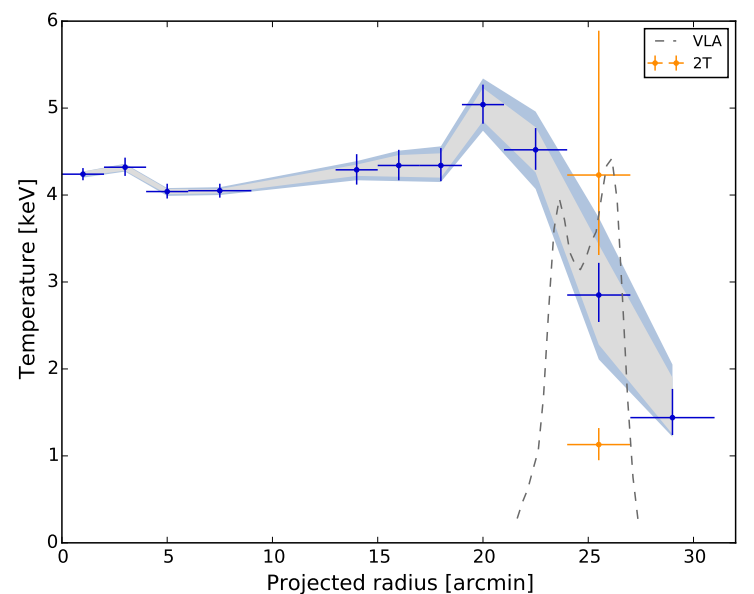

Fig. 5. Radial temperature profile for the western region. The gray and blue area represent the CXB and NXB systematic uncertainties. The orange points show the $2 \mathrm{~T}$ model temperatures at the western radio relic. The dashed gray line is the VLA radio radial profile.

been fitted simultaneously. We analyzed the NXB subtracted spectra with the normalization Norm, temperature $k T$ and metal abundance $Z$ for the inner region $r \leq 9^{\prime}$ as free parameters. The sky background components have been fixed to the values presented in Table 2.

The best-fit parameters are summarized in Table 3 . In general, we obtained a good fit for all regions (C-stat/d.o.f. $<1.2)$. The resulting radial temperature profile is shown in Fig. 5. It includes in gray and blue the systematic uncertainties due to the CXB fluctuation and NXB, respectively, as mentioned above. The radial profile shows an average temperature of $\sim 4 \mathrm{keV}$ in the central region of the cluster as found earlier by De Grandi \& Molendi (2002), Kawano et al. (2009) and Akamatsu et al. (2012). At $r=20^{\prime}$ the temperature increases slightly to $\sim 5 \mathrm{keV}$ and drops smoothly to $\sim 1.4 \mathrm{keV}$ beyond the western radio relic. Although there is a temperature decrease, there is not a clear discontinuity in the temperature at the radio relic. One possible explanation is that the annular region at $r=24^{\prime}-27^{\prime}$ contains gas from the pre and post relic region, which have two different temperatures. In order to investigate this aspect we included a second cie model in this region. As a result, we obtained two distinct temperatures: $k T_{1}=4.2 \pm 1.3 \mathrm{keV}$ and $k T_{2}=1.1 \pm 0.2 \mathrm{keV}$ (see orange points in Fig. 5) which are consistent with 


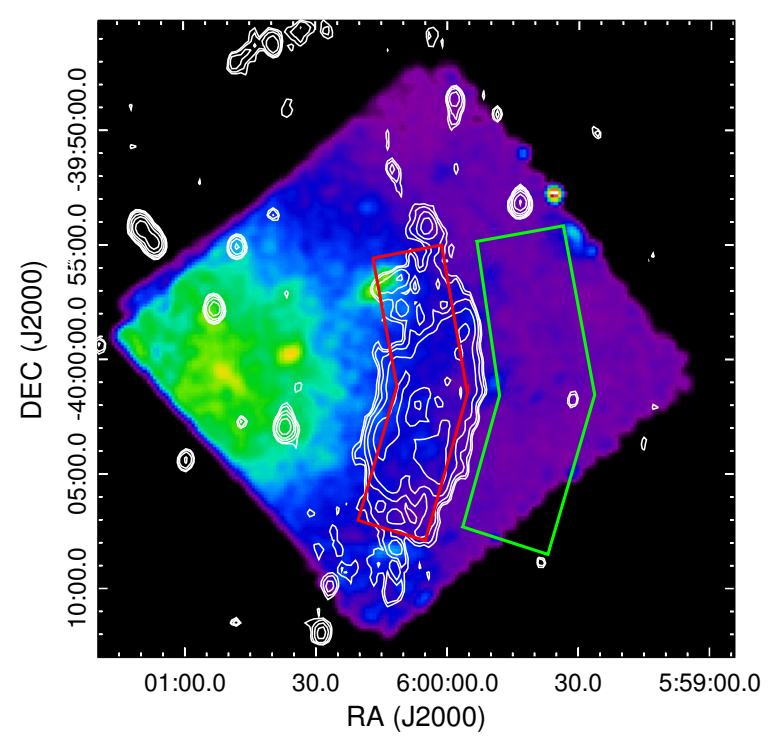

Fig. 6. A3376 $\mathrm{W} 1$ and $\mathrm{W} 2 \mathrm{NXB}$ subtracted images in the band $0.5-10 \mathrm{keV}$. The white contours correspond to VLA radio observations. The green and red polygonal regions are the pre and post-shock regions of the western radio relic, respectively.

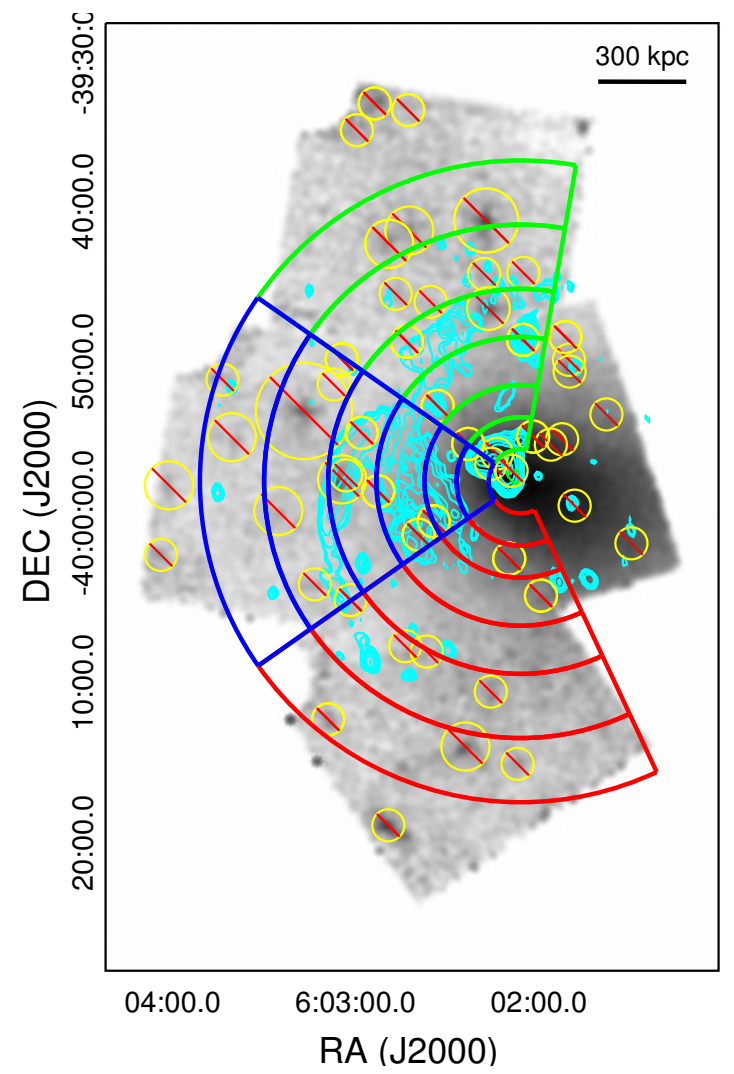

Fig. 7. A3376 center, north, east, and south images in the band $0.5-10 \mathrm{keV}$. The cyan contours represent VLA radio observations. The yellow circles represent the extracted points sources. The green (north), blue (east) and red (south) annular regions are used for the spectral analysis detailed in Tables 6, 5, and 7, respectively.

adjacent regions indicating the presence of multi-temperature structure within the extraction region. Therefore, this could be a preliminary indication of a temperature jump in the radio relic area and the possible presence of a shock front.
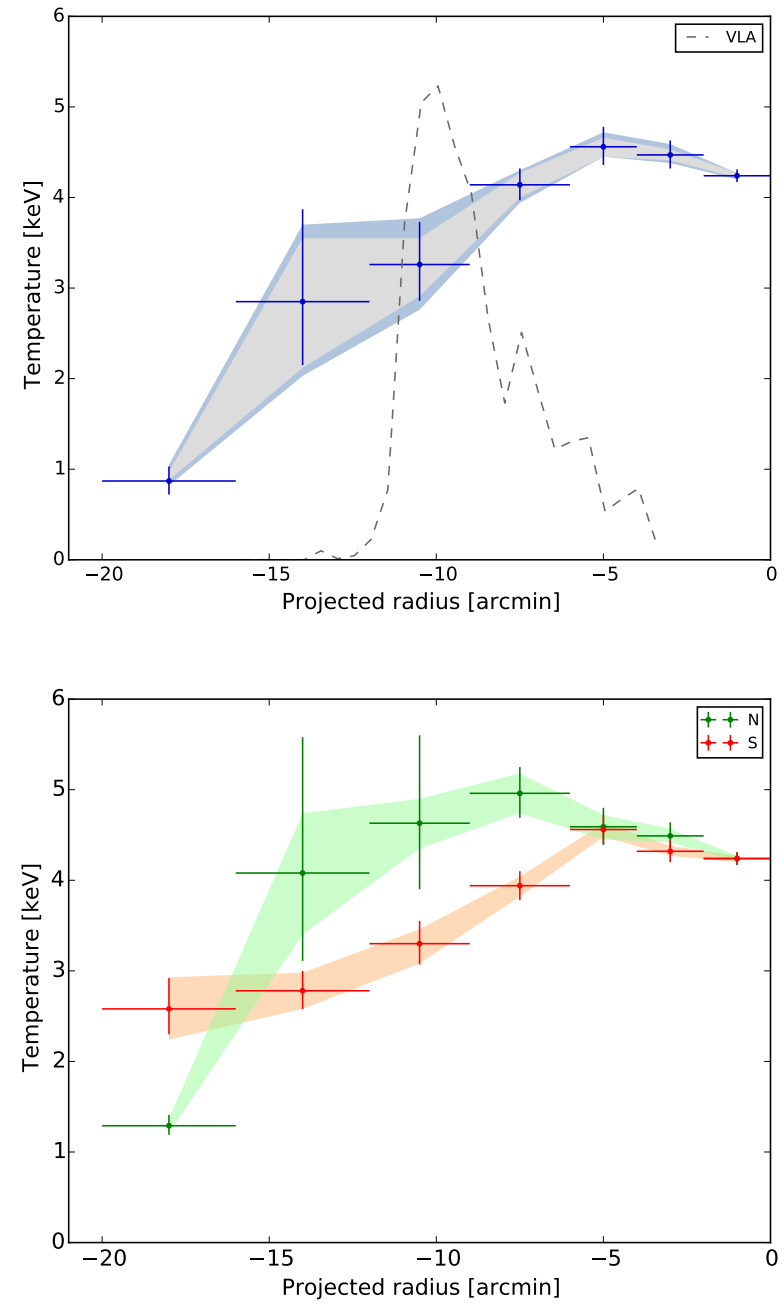

Fig. 8. Radial temperature profile for the eastern region (top is east and bottom is north and south). Top: Gray and blue areas represent the CXB and NXB systematic uncertainties. The dashed gray line is the VLA radio radial profile. Bottom: Green and red areas represent the $\mathrm{CXB}$ and NXB systematic uncertainties for north and south, respectively.

As a next step, we defined two additional regions in order to obtain the temperature upstream and downstream of the possible X-ray shock (Fig. 6). We introduced a separation of $\sim 1^{\prime}$ between pre and post-shock region to avoid a possible photon leakage from the brighter region due to limited PSF of XRT (Akamatsu et al. $2015,2017)$. The pre-shock region is located beyond the radio relic edge (the green polygonal region in Fig. 6) and the post-shock region is inside the radio relic edge (the red polygonal region in Fig. 6). The resulting best-fit parameters for these pre and postshock regions are shown in Table 4 and the spectrum of the postshock region is shown in Fig. 9. In this new analysis, the temperature shows a significant drop from $k T_{\text {post }}=4.22 \pm 0.26 \mathrm{keV}$ to $k T_{\text {pre }}=1.27 \pm 0.29 \mathrm{keV}$. These ICM temperatures of the pre and post-shock regions agree with previous Suzaku results $\left(k T_{\text {post }}=4.68_{-0.24}^{+0.42}\right.$ to $k T_{\text {pre }}=1.34 \pm 0.42 \mathrm{keV}$, Akamatsu et al. 2012). We discuss the shock properties related to the western radio relic in Sect. 4.3.

\subsection{Spectral analysis along the eastern region}

In the spectral analysis of the eastern region we have studied three different directions: N, E, and S, corresponding to the observations with the same names (see Table 1). We used 
I. Urdampilleta et al.: X-ray study of the double radio relic Abell 3376 with Suzaku
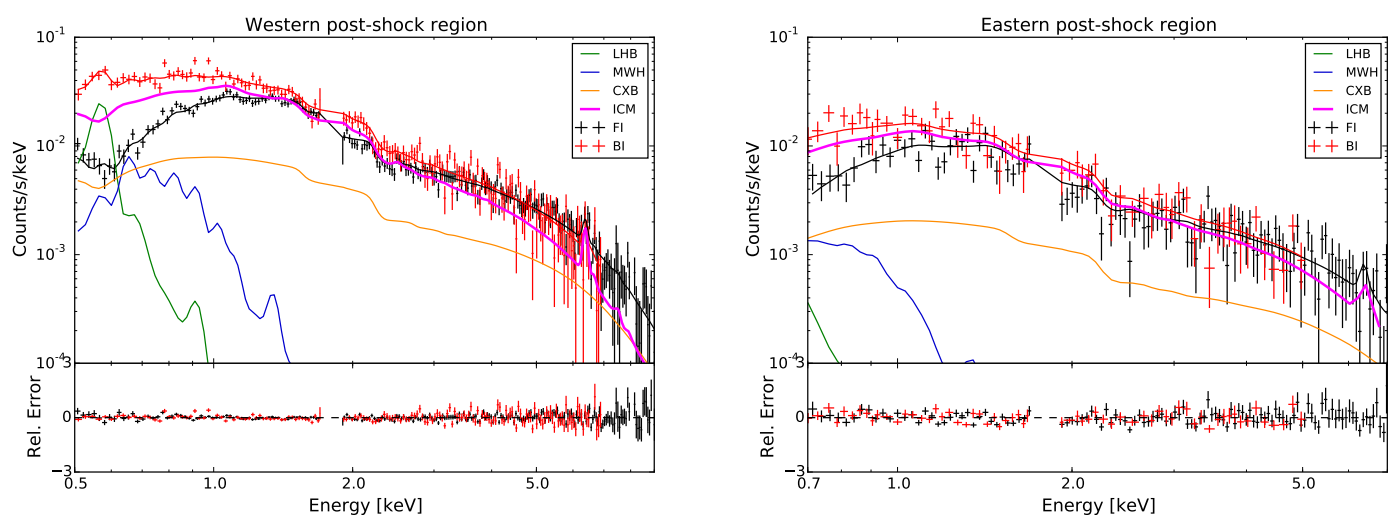

Fig. 9. Left: NXB-subtracted spectrum of western post-shock region in the $0.5-10 \mathrm{keV}$ band. Right: eastern post-shock spectrum in the $0.7-$ $7.0 \mathrm{keV}$ band. The FI (black) and BI (red) spectra are fitted with the ICM model together with the CXB and Galactic emission. The ICM is shown in magenta. The LHB, MWH and CXB are represented by green, blue and orange curves, respectively. The ICM, LHB, MWH, and CXB has been represented relative to the $\mathrm{BI}$ spectrum.

Table 5. Best-fit parameters for the center and east regions shown in Fig. 7.

\begin{tabular}{cccccc}
\hline \hline & Radius $\left(^{\prime}\right)$ & $k T(\mathrm{keV})$ & Norm $\left(10^{73} \mathrm{~m}^{-3}\right)$ & $Z\left(Z_{\odot}\right)$ & C-stat/d.o.f. \\
\hline \multirow{2}{*}{ Center } & $3.0 \pm 1.0$ & $4.47 \pm 0.16$ & $4.11 \pm 0.11$ & $0.33 \pm 0.06$ & $491 / 451$ \\
& $5.0 \pm 1.0$ & $4.56 \pm 0.21$ & $1.96 \pm 0.06$ & $0.25 \pm 0.07$ & $361 / 340$ \\
\hline C\&E & $7.5 \pm 1.5$ & $4.14 \pm 0.18$ & $0.95 \pm 0.03$ & $0.34 \pm 0.08$ & $661 / 630$ \\
\hline \multirow{3}{*}{ East } & $10.5 \pm 1.5$ & $3.26 \pm 0.44$ & $0.36 \pm 0.02$ & 0.3 (fixed) & $258 / 266$ \\
& $14.0 \pm 2.0$ & $2.85 \pm 0.86$ & $0.12 \pm 0.02$ & 0.3 (fixed) & $170 / 169$ \\
& $18.0 \pm 2.0$ & $0.87 \pm 0.16$ & $0.02 \pm 0.01$ & 0.3 (fixed) & $180 / 196$ \\
\hline
\end{tabular}

Table 6. Best-fit parameters for the center and north regions shown in Fig. 7.

\begin{tabular}{cccccc}
\hline \hline & Radius $\left(^{\prime}\right)$ & $k \mathrm{~T}(\mathrm{keV})$ & Norm $\left(10^{73} \mathrm{~m}^{-3}\right)$ & $Z\left(Z_{\odot}\right)$ & C-stat/d.o.f. \\
\hline \multirow{4}{*}{ Center } & $3.0 \pm 1.0$ & $4.49 \pm 0.15$ & $3.81 \pm 0.09$ & $0.38 \pm 0.06$ & $522 / 509$ \\
& $7.5 \pm 1.0$ & $4.59 \pm 0.21$ & $1.73 \pm 0.05$ & $0.40 \pm 0.09$ & $331 / 363$ \\
& $4.96 \pm 0.28$ & $0.94 \pm 0.03$ & $0.36 \pm 0.11$ & $343 / 327$ \\
\hline \multirow{4}{*}{ North } & $10.5 \pm 1.5$ & $4.63 \pm 0.85$ & $0.48 \pm 0.04$ & 0.3 (fixed) & $129 / 118$ \\
& $14.0 \pm 2.0$ & $4.08 \pm 1.24$ & $0.17 \pm 0.02$ & 0.3 (fixed) & $126 / 157$ \\
& $18.0 \pm 2.0$ & $1.29 \pm 0.11$ & $0.12 \pm 0.02$ & 0.3 (fixed) & $192 / 173$ \\
\hline
\end{tabular}

Table 7. Best-fit parameters for the center and south regions shown in Fig. 7.

\begin{tabular}{cccccc}
\hline \hline & Radius $\left(^{\prime}\right)$ & $k T(\mathrm{keV})$ & Norm $\left(10^{73} \mathrm{~m}^{-3}\right)$ & $Z\left(Z_{\odot}\right)$ & C-stat/d.o.f. \\
\hline \multirow{6}{*}{ Center } & $3.0 \pm 1.0$ & $4.32 \pm 0.12$ & $5.04 \pm 0.11$ & $0.38 \pm 0.06$ & $578 / 559$ \\
& $5.0 \pm 1.0$ & $4.56 \pm 0.16$ & $3.18 \pm 0.08$ & $0.47 \pm 0.07$ & $396 / 399$ \\
& $7.5 \pm 1.0$ & $3.94 \pm 0.16$ & $1.82 \pm 0.05$ & $0.25 \pm 0.07$ & $326 / 359$ \\
\hline \multirow{5}{*}{ South } & $10.5 \pm 1.5$ & $3.30 \pm 0.24$ & $0.66 \pm 0.03$ & 0.3 (fixed) & $386 / 370$ \\
& $14.0 \pm 2.0$ & $2.78 \pm 0.21$ & $0.44 \pm 0.02$ & 0.3 (fixed) & $271 / 251$ \\
& $18.0 \pm 2.0$ & $2.58 \pm 0.31$ & $0.25 \pm 0.02$ & 0.3 (fixed) & $204 / 213$ \\
\hline
\end{tabular}

annular regions, with the same centroid as the western region $\left(\mathrm{RA}=6^{\mathrm{h}} 02^{\mathrm{m}} 07 \mathrm{~s} .66, \quad\right.$ Dec $\left.=-39^{\circ} 57^{\prime} 42^{\prime \prime} \cdot 74\right)$, between $2^{\prime}-4^{\prime}$, $4^{\prime}-6^{\prime}, 6^{\prime}-9^{\prime}, 9^{\prime}-12^{\prime}, 12^{\prime}-16^{\prime}$ and $16^{\prime}-20^{\prime}$. Figure 7 shows green regions for $\mathrm{N}$, blue regions for $\mathrm{E}$ and red regions for $\mathrm{S}$. We fit the FI $(0.7-7.0 \mathrm{keV})$ and BI $(0.7-5.0 \mathrm{keV})$ spectra simultaneously. We applied the same criteria for the background components 


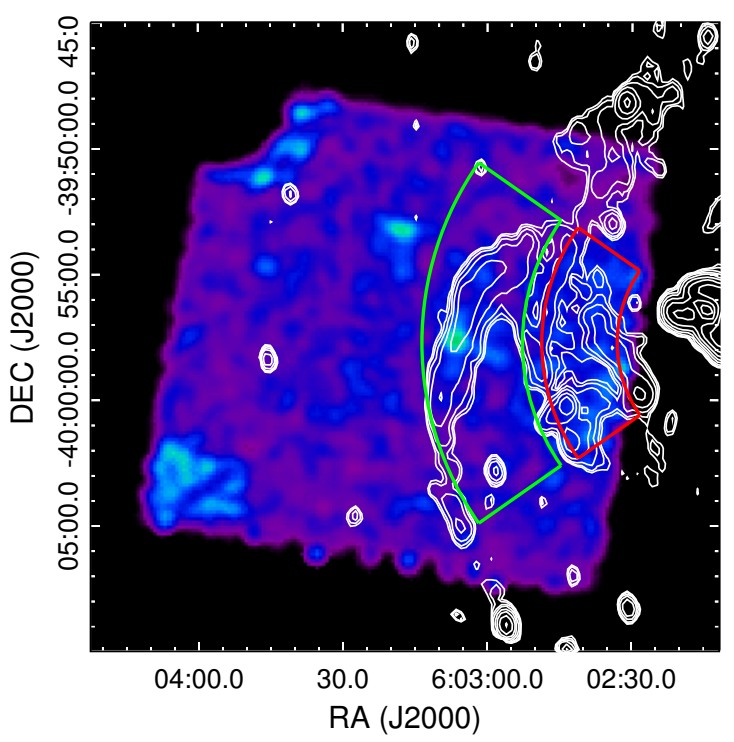

Fig. 10. A3376 East NXB subtracted images in the band $0.5-10 \mathrm{keV}$. The white contours correspond to VLA radio observations. The green and red annular regions are the pre and post-shock regions of the eastern and northern radio relic, respectively.

Table 8. Best-fit parameters for the pre- and post-shock regions at the east region shown in Fig. 10.

\begin{tabular}{cccc}
\hline \hline & $k T(\mathrm{keV})$ & Norm $\left(10^{72} \mathrm{~m}^{-3}\right)$ & C-stat/d.o.f. \\
\hline Post & $4.71 \pm 0.42$ & $11.7 \pm 0.1$ & $192 / 187$ \\
Pre & $3.31 \pm 0.44$ & $3.2 \pm 0.2$ & $182 / 173$ \\
\hline
\end{tabular}

and the ICM definition as explained above for the western regions. The best-fit parameters for east, north, and south are summarized in Tables 5, 6, and 7, respectively. In general we obtained a good fit with C-stat/d.o.f. $<1.1$ for E and S, and C-stat/d.o.f. $<1.25$ for N. Figure 8 shows the radial temperature profile for the three directions. In general, the statistical errors in our data are larger than or of the same order of magnitude as the systematic errors.

The radial temperature profile in the $\mathrm{E}$ direction shows a slight increase of the temperature at the center followed by a temperature gradient until $\sim 1 \mathrm{keV}$ in the cluster outer region. Motivated by a surface brightness discontinuity found at $r \sim 8^{\prime}$ explained in Sect. 4.2, we then analyzed a pre-shock (green annular) and post-shock (red annular) region (see Fig. 10). The best-fit parameters show a decrease from $k T_{\text {post }}=4.71 \mathrm{keV}$ to $k T_{\text {pre }}=3.31 \mathrm{keV}$ (Table 8). The spectrum of the postshock region is shown in Fig. 9. These ICM temperatures are consistent with the ones estimated in the eastern annular regions at $r=6-9^{\prime}$ and $r=9-12^{\prime}$.

For the $\mathrm{N}$ direction, we divided the annular regions in two $\left(\mathrm{N}_{\mathrm{a}}: 350-22.5^{\circ}\right.$ and $\left.\mathrm{N}_{\mathrm{b}}: 22.5-55^{\circ}\right)$ sections to investigate possible azimuthal differences. After the spectral analysis we could not find any significant difference between both sides. Therefore, we have analyzed the full annular regions as shown in Fig. 7. The temperature increases up to $\sim 5 \mathrm{keV}$ at $r=7.5^{\prime}$ and decreases till $\sim 1 \mathrm{keV}$ in the cluster outskirts. We investigated pre and postshock regions at $r=8^{\prime}$, similar to the case of the eastern direction to analyze the extent of the eastern shock, and there are no signs of a temperature drop.
In the $\mathrm{S}$ direction, we obtain a radial temperature profile with a central temperature of $\sim 4 \mathrm{keV}$ and a smooth decrease down to the cluster peripheries. It follows the predicted trend for relaxed galaxy clusters as described by Reiprich et al. (2013). We discuss this behavior in more detail in Sect. 4.1.

\section{Discussion of spectral analysis}

\subsection{ICM temperature profile}

The main growing mechanism of galaxy clusters includes the accretion and merging of the surrounding galaxy groups and subclusters. These processes are highly energetic and turbulent, being able to modify completely the temperature structure of the ICM (Markevitch \& Vikhlinin 2007). Therefore, this temperature structure contains the signatures of how the cluster has evolved, providing relevant information on the growth and heating history.

There are fewer studies about merging galaxy clusters and the impact of the above events to the ICM temperature structure then about relaxed clusters. A recent compilation of Suzaku observations shows the temperature profile up to cluster outskirts (Reiprich et al. 2013). That review shows that relaxed clusters have a similar behavior near $r_{200}$ and that the temperature can smoothly drop by a factor of three at the periphery. Moreover, these Suzaku data are consistent with the ICM temperature model of relaxed galaxy clusters proposed by Burns et al. (2010).

Burns et al. (2010) obtained this "universal" profile model based on N-body plus hydrodynamic simulations for relaxed clusters. The scaled temperature profile as a function of normalized radius is given by:

$\frac{T}{T_{\text {avg }}}=A\left[1+B\left(\frac{r}{r_{200}}\right)\right]^{\beta}$,

where the best-fit parameters are $A=1.74 \pm 0.03$, $B=0.64 \pm 0.10$ and $\beta=3.2 \pm 0.4 . T_{\text {avg }}$ is the average X-ray weighted temperature between $0.2-1.0 r_{200}$.

In Fig. 11 we compare Burns' radial profile with the radial temperature profile of A3376 normalized with $\langle k T\rangle=4.2 \mathrm{keV}$ $\left(\left\langle k T_{x}\right\rangle\right.$ up to $0.3 r_{200}$, (Reiprich et al. 2009) and $r_{200} \sim 1.76 \mathrm{Mpc}$ derived from Henry et al. (2009). The western direction shows an enhancement of the temperature compared with the relaxed profile and a sharp drop close to $\sim 0.7 r_{200}$. This is a hint for the presence of a shock front and shock heating of the ICM at these radii. A similar, but less pronounced, behavior is found for the north and east, being the temperature excess higher for the north. In both cases, the temperature shows a decrease around $\sim 0.3 r_{200}$, where the eastern radio relic is located, with a steeper temperature profile than relaxed clusters. In the north, the large statistical errors and the weakness of the signal limit the detection of a temperature jump, and therefore, the possible evidence for a shock front. Deeper observations are needed to constrain this temperature structure in more detail. On the other hand, the southern direction seems to follow the profile of relaxed clusters. This is expected because there is no radio emission in this direction.

\subsection{X-ray surface brightness profiles}

In order to confirm the evidence of bow shocks at the western and eastern regions, we analyzed the radial X-ray surface brightness profile from the center of A3376 using the XMM-Newton observations. The SB was determined in the $0.3-2.0 \mathrm{keV}$ band 


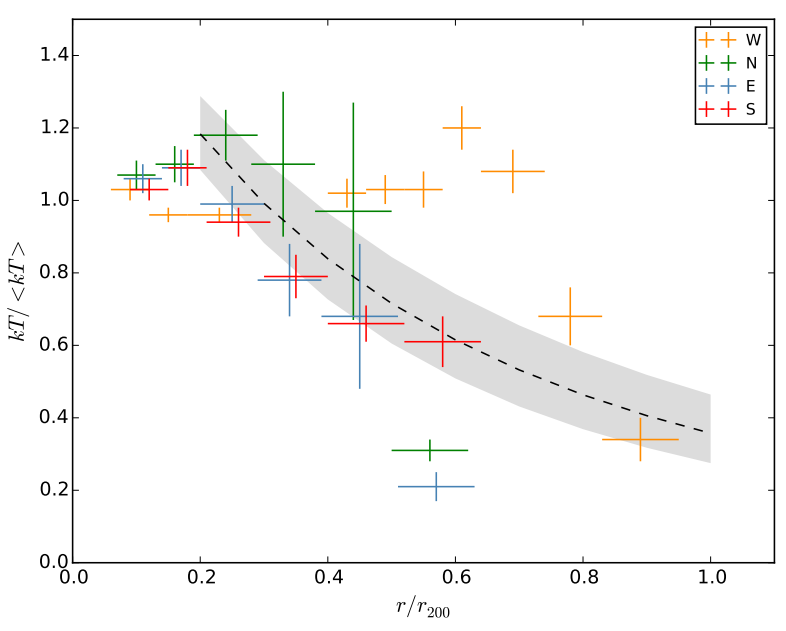

Fig. 11. Normalized radial temperature profile of A3376 compared with relaxed clusters. The dashed line represents the Burns et al. (2010) universal profile and the gray area shows its standard deviation. The orange, green, blue and red crosses are the scaled data for the west, north, east, and south directions, respectively. We have shifted these scaled data of the different directions in $r / r_{200}$ for clarity purpose.

excluding the Al line at $\sim 1.5 \mathrm{keV}$. Point sources with a flux higher than $S_{\mathrm{c}}=10^{-17} \mathrm{~W} \mathrm{~m}^{-2}$ were removed (for more details see Sect. 3.2) and the X-ray image was corrected for exposure and background. The sky background components derived from Q0551-3637 (see Table 2) have been adopted together with the instrumental background for the background correction. The surface brightness profile was extracted in circular pie shaped sectors (see sectors in Fig. 12) and fitted with PROFFIT v1.4 (Eckert et al. 2011). We adopted a broken power-law density profile to describe the SB profile. Therefore, assuming spherical symmetry, the density distribution is given by

$$
\begin{cases}n_{2}(r)=n_{0}\left(\frac{r}{r_{\mathrm{sh}}}\right)^{\alpha_{2}} & r \leq r_{\mathrm{sh}} \\ n_{1}(r)=\frac{1}{C} n_{0}\left(\frac{r}{r_{\mathrm{sh}}}\right)^{\alpha_{1}} & r>r_{\mathrm{sh}}\end{cases}
$$

where $n_{0}$ is the model density normalization, $\alpha_{1}$ and $\alpha_{2}$ are the power-law indices, $r$ is the radius from the center of the sector and $r_{\mathrm{sh}}$ is the shock putative distance. At the location of the SB discontinuity $n_{2}$, the post-shock (downstream) density is higher by a factor of $C=n_{2} / n_{1}$ compared with $n_{1}$, pre-shock (upstream) density. This factor $C$ is known as the compression factor. In the X-ray SB fitting, we left all these model parameters free to vary.

The radial SB profile across the western radio relic is shown in Fig. 13. The SB was accumulated in circularly shaped sectors to match the outer edge of the radio relic from an angle of $230^{\circ}$ to $280^{\circ}$ (measured counter-clockwise) for $r=21-28^{\prime}$. The data were rebinned to reach a minimum signal-to-noise ratio $(\mathrm{S} / \mathrm{N})$ of four. The best-fitting broken power-law model is shown as the blue line with C-stat/d.o.f. 1.2. It presents a break inside the radio relic at $r_{\mathrm{sh}} \sim 23^{\prime}$ (measured from the X-ray emission peak centroid) and the compression factor is $C=1.9 \pm 0.4$. If we apply the PSF modeling of XMM-Newton as described in Appendix C of Eckert et al. (2016a) for a more accurate estimate of the density profile, then the density jump increases to $C=2.1 \pm 0.6$. We have additionally evaluated different elliptical shaped sectors to adjust them to the relic shape. They have

\footnotetext{
7 http://www.isdc.unige.ch/ deckert/newsite/Proffit. html
}

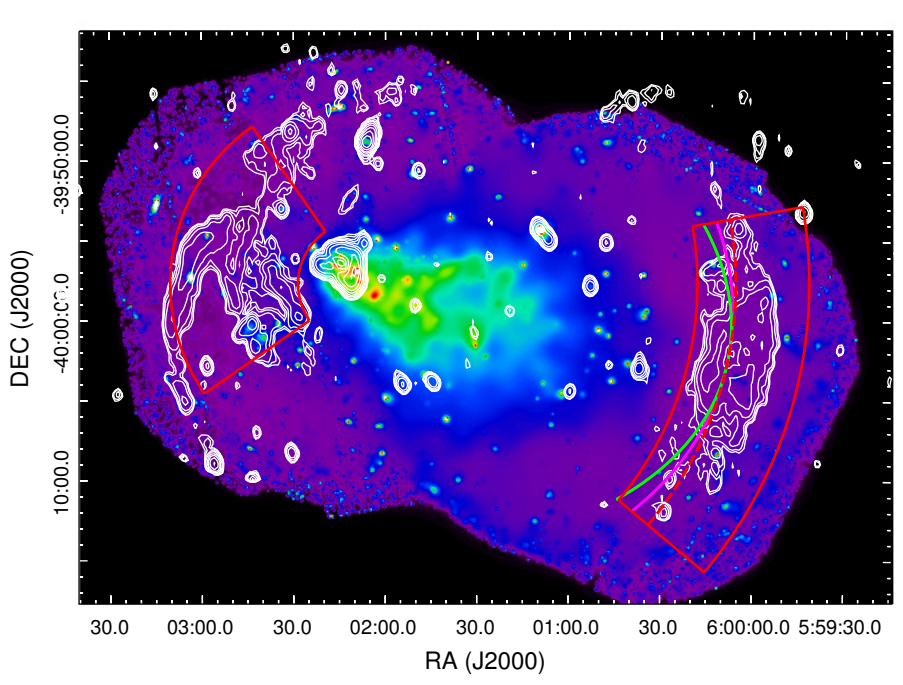

Fig. 12. XMM-Newton image in the $0.5-10 \mathrm{keV}$ band of A3376. The white contours correspond to VLA radio observations. The red sectors are used to extract the X-ray SB profile in the $\mathrm{E}$ and $\mathrm{W}$ directions. The dashed red line represents the circular $(\varepsilon=0)$ shaped sector used for the SB profiles. The magenta and green lines are the elliptical shaped sectors with $\varepsilon=0.60$ and $\varepsilon=0.73$, respectively.

eccentricities of $\varepsilon=0.6$ and $\varepsilon=0.73$. The compression factors obtained in both cases are lower than for the circular sector, $C=$ $1.7 \pm 0.3$ and $C=1.5 \pm 0.4$, respectively. The different radii for the SB discontinuity compared with the temperature jump could be caused by the Suzaku PSF $\sim 2$.

The obtained value of $C=2.1 \pm 0.6$ is consistent within the $1 \sigma$ uncertainty bounds with the value in Table $9, C=2.9 \pm 0.3$, although it shows a slightly lower value. This could possibly be explained because of the difficulty to model the multi-component background in the lack of any sky region where it cannot be spatially separated from the ICM. Therefore, the background modeling can play also an important role in the SB profiles located at the outskirts. Future observations with the Athena satellite could provide an explanation to this issue.

We obtained the radial SB profile along the E direction for circular sectors between $35^{\circ}$ to $125^{\circ}$ with the center at the X-ray emission peak. The data were rebinned to have a minimum $S / N \sim 8$. For radii larger than $12^{\prime}$ the $\mathrm{SB}$ emission is low and is background dominated. For this reason, we selected the range $4-12^{\prime}$ for the fitting. The best-fitting broken power-law model is shown in Fig. 14 as a blue line with C-stat/d.o.f. 1.4. The SB profile contains an edge at $r \sim 8^{\prime}$ and the compression factor is $C=1.9 \pm 0.5$, after applying the same PSF modeling as for the western SB profile. Because the radial profile of the radio relic azimutally averages various features (see VLA radio contours in Figs. 10 and 12), this edge appears to be located ahead of a secondary peak in the radio relic profile. It seems to be associated to the "notch" described by Paul et al. (2011) and Kale et al. (2012).

\subsection{Shock jump conditions and properties}

The density (surface brightness) and temperature discontinuities found along the western and eastern directions form evidence for a shock front co-spatial with the western relic (Fig. 13 and Table 4) and the "notch" radio structure in the east (Fig. 14 and Table 8), respectively. Here, we calculate the shock properties in the western and eastern directions based on our Suzaku 


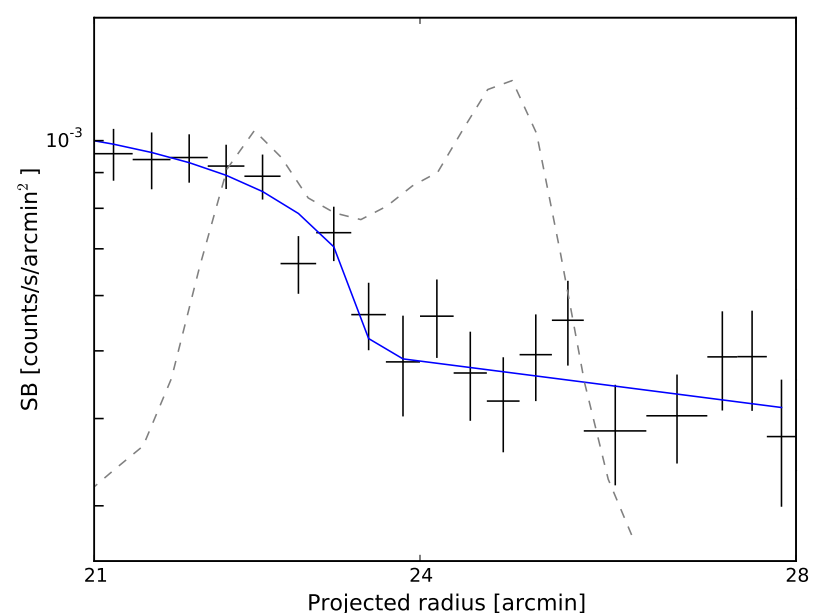

Fig. 13. Radial X-ray surface brightness profile across the western radio relic in the $0.3-2.0 \mathrm{keV}$ band using $X M M$-Newton observations The profile is corrected for vignetting and background level, and point sources have been removed. The solid blue curve is the best-fit model and the gray dashed line is the VLA scaled radio emission. The data were rebinned to reach a minimum $\mathrm{S} / \mathrm{N}$ of four and $\mathrm{C}$-stat/d.o.f. $\sim 1.2$.

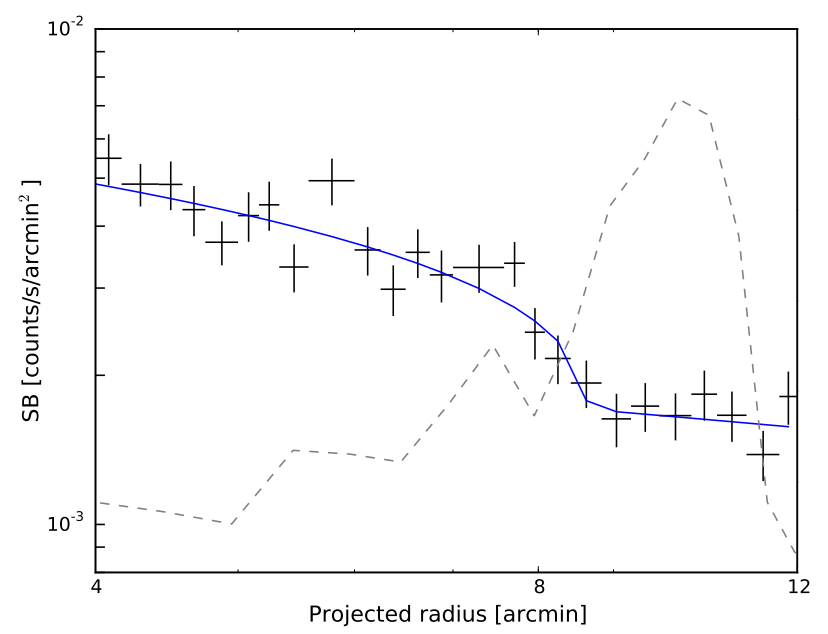

Fig. 14. Radial $X$-ray surface brightness profile across the eastern radio relic in the $0.3-2.0 \mathrm{keV}$ band using XMM-Newton observations. Same as Fig. 13, with a minimum $\mathrm{S} / \mathrm{N}$ of eight and $\mathrm{C}$-stat/d.o.f. $~ 1.4$.

observations. The Mach number $(\mathcal{M})$ and compression factor (C) can be assessed from the Rankine-Hugoniot jump condition (Landau \& Lifshitz 1959) assuming that all of the dissipated shock energy is thermalized and the ratio of specific heats (the adiabatic index) is $\gamma=5 / 3$ :

$\frac{T_{2}}{T_{1}}=\frac{5 \mathcal{M}^{4}+14 \mathcal{M}^{2}-3}{16 \mathcal{M}^{2}}$,

$C=\frac{n_{2}}{n_{1}}=\frac{4 \mathcal{M}^{2}}{\mathcal{M}^{2}+3}$,

where $n$ is the electron density, and the indices 2 and 1 corresponds to post-shock and pre-shock regions, respectively. Table 9 shows the Mach numbers and compression factors derived from the observed temperature jumps. The Mach numbers are in good agreement with the simulations of Machado \& Lima Neto (2013) and $\mathcal{M}_{\mathrm{W}}$ is consistent with previous Suzaku results obtained by Akamatsu et al. $(2012)\left(\mathcal{M}_{\mathrm{W}}=3.0 \pm 0.5\right)$.
The Mach numbers estimated from the surface brightness discontinuities described in the previous sections are $\mathcal{M}_{\mathrm{W}_{\mathrm{SB}}} \sim$ 1.3-2.5 and $\mathcal{M}_{\mathrm{E}_{\mathrm{SB}}} \sim 1.7 \pm 0.4$. Both values are consistent with the value present in Table 9 within $1 \sigma$ error. The presence of shocks with $\mathcal{M} \gtrsim 3.0$ is uncommon in galaxy clusters, only four other clusters have been found with such a high Mach number ("El Gordo", Botteon et al. 2016b; A665, Dasadia et al. 2016a; CIZA J224.8+5301, Akamatsu et al. 2015; "Bullet", Shimwell et al. 2015).

The sound speed at the pre-shock regions is $c_{\mathrm{s}, \mathrm{W}} \sim$ $570 \mathrm{~km} \mathrm{~s}^{-1}$ and $c_{\mathrm{s}, \mathrm{E}} \sim 940 \mathrm{~km} \mathrm{~s}^{-1}$, assuming $c_{\mathrm{s}}=$ $\sqrt{\gamma k T_{1} / \mu m_{p}}$ where $\mu=0.6$. The shock propagation speeds $v_{\text {shock }}=\mathcal{M} \cdot c_{\mathrm{s}}$ for the western and eastern directions are $v_{\text {shock, } \mathrm{W}} \sim 1630 \pm 220 \mathrm{~km} \mathrm{~s}^{-1}$ and $v_{\text {shock,E }} \sim 1450 \pm 150 \mathrm{~km} \mathrm{~s}^{-1}$, respectively. These velocities are consistent with previous work for A3376 W (Akamatsu et al. 2012). However, the shock velocities are smaller than in other galaxy clusters with $\mathcal{M} \sim 2-3$ as the Bullet cluster $\left(4500 \mathrm{~km} \mathrm{~s}^{-1}\right.$, Markevitch et al. 2002), CIZA J2242.8+530 (2300 $\mathrm{km} \mathrm{s}^{-1}$, Akamatsu et al. 2017) and A520 (2300 $\mathrm{km} \mathrm{s}^{-1}$, Markevitch et al. 2005).

We have compiled the shock velocities, $v_{\text {shock }}$, for several merging galaxy clusters as shown in Fig. 15. Black points represent data taken directly from the literature and the gray points are calculated from $\mathcal{M}$ and $k T_{1}$. Blue and red crosses are the western and eastern shock velocities, respectively. Figure 15 shows shock velocities as a function of average temperature of the system. To investigate the origin of driving force of the shock structure, we have examined a prediction from self-similar relationship: $v_{\text {shock }}$ $=A *\langle k T\rangle^{(3 / 2)}+B$, where $A=70 \pm 16$ and $B=550 \pm 270$, see orange line. As we expected, most of samples can be explained with this formula, which means that the main driving force of the merging activity is the gravitational potential of the system and no additional physics. Further sample of shocks and proper gravitational mass estimates will enable us to extend this type of examination.

\subsection{Mach number from $X$-ray and radio observations}

After the first confirmation by Finoguenov et al. (2010) of a clear correlation between X-ray shock fronts and radio relics in A3667, many other observations have revealed a possible relation between them (Macario et al. 2011; Mazzota et al. 2011; Akamatsu \& Kawahara 2013). As explained in the introduction, the X-ray shock fronts may accelerate charged particles up to relativistic energies via diffusive shock acceleration (DSA, Drury 1983; Blandford \& Eichler 1987), which in the presence of a magnetic field can generate synchrotron emission. The acceleration efficiency of this mechanism is low for shocks with $\mathcal{M}<10$ and might not be sufficient to produce the observed radio spectral index (Kang et al. 2012; Pinzke et al. 2013). Therefore, alternative scenarios have been proposed like the presence of a fossil population of nonthermal electrons and re-acceleration of these electrons by shocks (Bonafede et al. 2014; van Weeren et al. 2017) or the electron re-acceleration by turbulence (Fujita et al. 2015; Kang 2017).

DSA is based on first order Fermi acceleration, considering that there is a stationary and continuous injection, which accelerates relativistic electrons following a power-law spectrum $n(E) \mathrm{d} E \sim E^{-p} \mathrm{~d} E$ with $p=(C+2) /(C-1), \alpha=-(p-1) / 2$ where $p$ is the power-law index and $\alpha$ is the radio spectral index for $S_{v} \propto v^{\alpha}$. The radio Mach number can be calculated from the injection spectral index as

$\mathcal{M}_{R}^{2}=\frac{2 \alpha-3}{2 \alpha+1}$. 
Table 9. Shock properties at the western and eastern radio relics.

\begin{tabular}{cccccccc}
\hline \hline & $\begin{array}{c}T_{2} \\
(\mathrm{keV})\end{array}$ & $\begin{array}{c}T_{1} \\
(\mathrm{keV})\end{array}$ & $\begin{array}{c}\text { Mach No. } \\
\mathcal{M}^{a}\end{array}$ & $\begin{array}{c}v_{\text {shock }} \\
\left(\mathrm{km} \mathrm{s}^{-1}\right)^{b}\end{array}$ & $\begin{array}{c}\text { Compression } \\
C^{c}\end{array}$ & $\begin{array}{c}\text { Power-law slope } \\
p^{d}\end{array}$ & $\begin{array}{c}\text { Spectrum index } \\
\alpha^{e}\end{array}$ \\
\hline $\mathrm{W}$ & $4.22 \pm 0.26$ & $1.27 \pm 0.29$ & $2.8 \pm 0.4$ & $1630 \pm 220$ & $2.9 \pm 0.3$ & $2.58 \pm 0.22$ & $-0.79 \pm 0.11$ \\
$\mathrm{E}$ & $4.71 \pm 0.42$ & $3.31 \pm 0.44$ & $1.5 \pm 0.1$ & $1450 \pm 150$ & $1.8 \pm 0.1$ & $4.92 \pm 0.85$ & $-1.96 \pm 0.43$ \\
\hline
\end{tabular}

Notes. ${ }^{(a)} \mathcal{M}$ is obtained from Eq. (6). ${ }^{(b)} v_{\text {shock }}=\mathcal{M} \cdot c_{\mathrm{s}}, c_{\mathrm{s}}=\sqrt{\gamma k T_{1} / \mu m_{p}} \cdot{ }^{(c)} C$ from Eq. (7). ${ }^{(d)} p=(C+2) /(C-1) .{ }^{(e)} \alpha=-(p-1) / 2$.

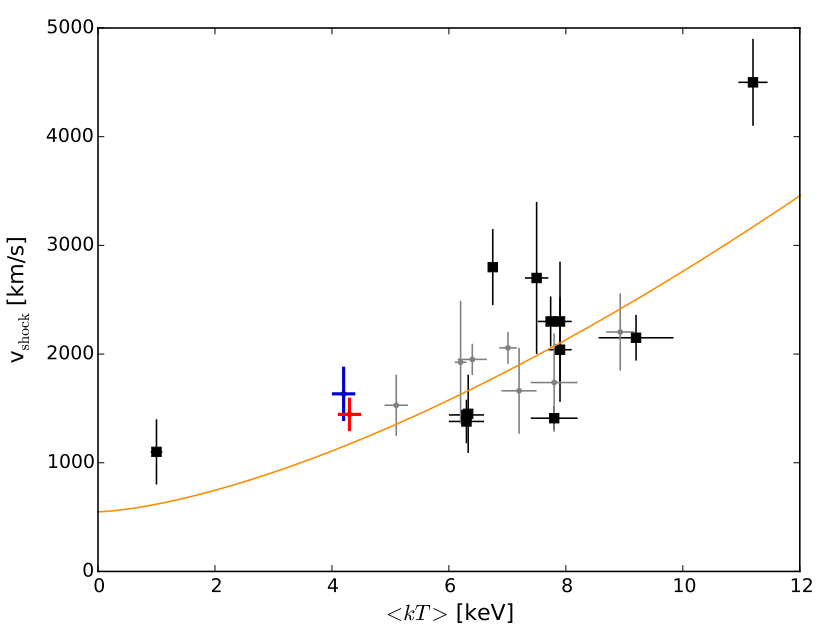

Fig. 15. $v_{\text {shock }}$ correlation with $\langle k T\rangle$. The orange line is the best-fit for $v_{\text {shock }}=A *\langle k T\rangle^{(3 / 2)}+B$. Black crosses represent data taken directly from the literature (Akamatsu \& Kawahara 2013; Akamatsu et al. 2015, 2017; Dasadia et al. 2016a; Eckert et al. 2016b; Markevitch et al. 2002, 2005; Russell et al. 2012, 2014; Sarazin et al. 2016). Gray points are the calculated data from $\mathcal{M}$ and $k T_{1}$ (Botteon et al. 2016a; Bourdin et al. 2013; Macario et al. 2011; Ogrean \& Brüggen 2013; Owers et al. 2014; Sarazin et al. 2016; Trasatti et al. 2015) as explained in the text. Blue and red crosses correspond to the $\mathrm{W}$ and $\mathrm{E}$ shocks of $\mathrm{A} 3376$ as obtained in this work, respectively.

For A3376 two radio observations exist in addition to the Very Large Array (VLA) observations by Bagchi et al. (2006): Giant Metrewave Radio Telescope, GMRT, 150 and $325 \mathrm{MHz}$ : Kale et al. (2012) and Murchison Widefield Array, MWA, 80-215 MHz: George et al. (2015). Kale et al. (2012) describe in detail the morphology of the $\mathrm{E}$ and $\mathrm{W}$ relics. They consider the DSA mechanism assuming that the synchrotron and inverse Compton losses have not affected the spectrum. Therefore, for the injected spectral index calculation they use the flattest spectrum in the outer edge of the spectral index map for 325-1400 $\mathrm{MHz}$. Their results are $\alpha_{\mathrm{E}}=-0.70 \pm 0.15$, which implies $\mathcal{M}_{\mathrm{E}}=$ $3.31 \pm 0.29$; and $\alpha_{\mathrm{W}}=-1.0 \pm 0.02$ with $\mathcal{M}_{\mathrm{W}}=2.23 \pm 0.40$. In this case, there are slight differences between the Mach number assessed from the X-ray and radio observations. George et al. (2015) have derived the integrated spectral indices of the eastern and western relic using the GMRT, MWA and VLA observations (see their Fig. 3) obtaining $\alpha_{\mathrm{E}}=-1.37 \pm 0.08$ and $\alpha_{\mathrm{W}}=-1.17 \pm 0.06$, which give the Mach numbers, assuming $\alpha_{\text {int }}=\alpha_{\text {inj }}-0.5, \mathcal{M}_{\mathrm{E}}=2.53 \pm 0.23$ and $\mathcal{M}_{\mathrm{W}}=3.57 \pm$ 0.58 , respectively. However, due to the low angular resolution of MWA, they can only resolve the integrated spectral index and not the injected one. For this reason, we have decided not to use the George et al. (2015) results for this study.

The differences between $\mathcal{M}_{\mathrm{R}}$ and $\mathcal{M}_{\mathrm{X}}$ are not only present in this galaxy cluster. Figure 16 includes several systems with similar behavior to A3376 (see Table 10), which is shown by a blue cross for A3376 W. A3376 E is not included because the spectral index reported by the radio observations refers to the elongated radio relic and not to the 'notch' structure. The orange crosses represent radio data obtained with LOFAR. $\mathcal{M}_{\mathrm{X}}$ of these clusters is derived from the temperature jump and $\mathcal{M}_{\mathrm{R}}$ is calculated using Eq. (8) with the injection spectral index $\alpha_{\text {inj. }}$ In addition, some other clusters as A521 (Bourdin et al. 2013; Giacintucci et al. 2008), A2034 (Owers et al. 2014; Shimwell et al. 2016) or 'El Gordo' (Botteon et al. 2016b; Lindner et al. 2014); present evidence for a shock front based on the surface brightness jump. In order to remain consistent regarding the method used to derive $\mathcal{M}$ (in our case, the temperature jump), we do not include these measurements in Fig. 16. We note that Mach numbers based on the SB jump seem to be smaller than those based on temperature jump (for more details, see Sarazin et al. 2016).

Akamatsu et al. (2017) discuss several possible reasons for the Mach number discrepancies in X-rays and radio. In this study we focus on the radio ageing effect (Pacholczyk 1973; Miniati 2002; Stroe et al. 2014), which can lead to lower radio $\mathcal{M}$ compared to X-rays. The electron ageing effect takes places when the relativistic electrons lose their energy via radiative cooling or inverse-Compton scattering after the shock passage in approximately less than $\sim 10^{7}-10^{8}$ years, which is shorter than the shock life time. As a consequence, the radio spectrum becomes steeper and the integrated spectral index decreases from $\alpha_{\text {int }}$ to $\alpha_{\text {inj }}-0.5$ in DSA model (Pacholczyk 1973; Miniati 2002). Only measurements with high angular resolution and low-frequency are able to measure $\alpha_{\text {inj }}$ directly. As a consequence, the calculation of $\mathcal{M}_{\mathrm{R}}$ might be underestimated. Moreover, it is thought that the simple relationship of $\alpha_{\text {int }}=\alpha_{\text {inj }}-0.5$ most likely does not hold for the relics case (Kang \& Ryu 2015, 2016). In the case of A3376, Kale et al. (2012) showed that the spectral distribution steepens from the outer to the inner edge in the frequency range $325-1400 \mathrm{MHz}$. However, to avoid the ageing effect they consider only the flattest spectral index as $\alpha_{\text {inj }}$ at the outer edge.

We calculated the distance free of ageing effect as $d=$ $t_{\text {loss }} \times v_{\text {gas }}$, where $t_{\text {loss }}$ is the cooling time for relativistic electrons and $v_{\text {gas }}=v_{\text {shock }} / C$ is the gas velocity. We estimated $t_{\text {loss }} \sim$ $10-5 \times 10^{7}$ yr from Eq. (14) of Kang et al. (2012):

$t_{\text {loss }} \approx 8.7 \times 10^{8} \mathrm{yr}\left(\frac{B^{1 / 2}}{B_{\text {eff }}^{2}}\right)\left(\frac{v_{\text {obs }}}{1 \mathrm{GHz}}\right)^{-1 / 2}(1+\mathrm{z})^{-1 / 2}$,

where we assumed a magnetic field of $B \sim 1 \mu \mathrm{G}$ and $v_{\text {obs }}=$ $325 \mathrm{MHz}-1.4 \mathrm{GHz}$. The effective magnetic field is $B_{\text {eff }}^{2}=$ $B^{2}+B_{\mathrm{CBR}}^{2}$, which includes the equivalent strength of the cosmic background radiation like $B_{\mathrm{CBR}}=3.24 \mu \mathrm{G}(1+z)^{2}$. The gas velocity is $v_{\text {gas, }} \sim 564 \mathrm{~km} \mathrm{~s}^{-1}$ for the west. Therefore, the distance free of the ageing effects is $d_{\mathrm{W}} \sim 58-28 \mathrm{kpc}$. In the case of assuming a five times higher magnetic field, $B \sim 5 \mu \mathrm{G}$, $t_{\text {loss }} \sim 4 \times 10^{7} \mathrm{yr}(20 \%$ lower than with $B \sim 1 \mu \mathrm{G})$ and for $B \sim 0.2 \mu \mathrm{G} t_{\text {loss }} \sim 2 \times 10^{7} \mathrm{yr}(60 \%)$, for $v_{\text {obs }}=1.4 \mathrm{GHz}$. Therefore, the assumptions of $B \sim 1 \mu \mathrm{G}$ seems the most conservative 


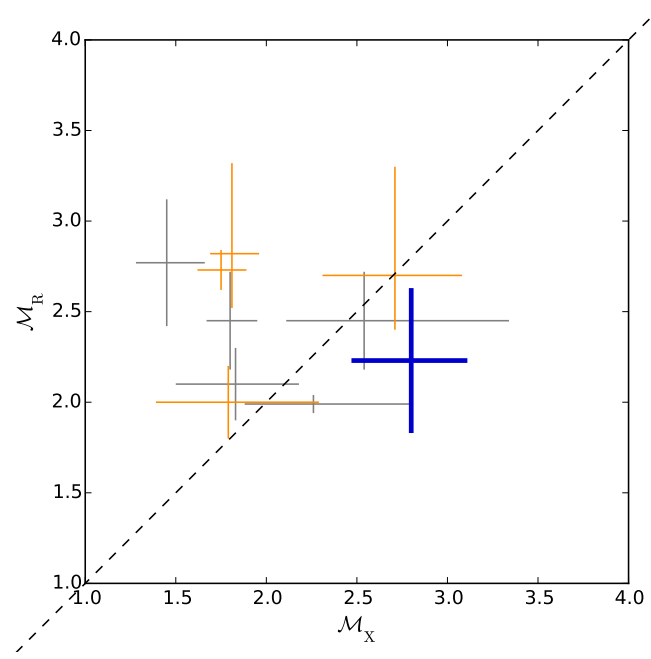

Fig. 16. Comparison between Mach number derived from radio observations $\left(\mathcal{M}_{R}\right)$ based on the radio spectral index and the X-ray observations $\left(\mathcal{M}_{\mathrm{X}}\right)$. The results for $\mathrm{A} 3376 \mathrm{~W}$ is represented by the blue cross. The gray crosses show data for others clusters (see Table 10), orange crosses show recent radio observations done with LOFAR references. The black dashed line is the linear correlation used as reference.

Table 10. The cluster sample for our $\mathcal{M}_{\mathrm{X}}$ and $\mathcal{M}_{\mathrm{R}}$ comparison.

\begin{tabular}{ccc}
\hline \hline Cluster & $\mathcal{M}_{\mathrm{X}}^{\dagger}$ & $\mathcal{M}_{\mathrm{R}}$ \\
\hline A2256* & $1.8 \pm 0.1(1)$ & $2.7 \pm 0.1(2)$ \\
A2255 NE & $1.5 \pm 0.2(3)$ & $2.8 \pm 0.4(4)$ \\
A115 & $1.8 \pm 0.3(5)$ & $2.1 \pm 0.2(5)$ \\
Sausage N $^{*}$ & $2.7 \pm 0.4(6)$ & $2.7 \pm 0.5(8)$ \\
Sausage S$^{*}$ & $1.8 \pm 0.5(7)$ & $2.0 \pm 0.2(8)$ \\
A3667 NW & $2.5_{-0.4}^{+0.8(9)}$ & $2.5 \pm 0.3(10)$ \\
A3667 SE & $1.8 \pm 0.1(11)$ & $2.5 \pm 0.3(10)$ \\
Coma & $2.3 \pm 0.5(12)$ & $1.99 \pm 0.05(13)$ \\
Toothbrush & $1.8 \pm 0.2(14)$ & $2.8 \pm 0.4(15)$ \\
A3376 W & $2.8 \pm 0.4$ & $2.2 \pm 0.4(16)$ \\
\hline
\end{tabular}

Notes. ${ }^{\left({ }^{*}\right)}$ Radio observations with LOFAR. ${ }^{(\dagger)} \mathcal{M}_{\mathrm{X}}$ has been calculated as described by Sarazin et al. (2016).

References: (1) Trasatti et al. (2015); (2) van Weeren et al. (2012); (3) Akamatsu et al. (2017); (4) Pizzo \& de Bruyn (2009); (5) Botteon et al. (2016a); (6) Akamatsu et al. (2015); (7) Storm et al (2018); (8) Hoang et al. (2017); (9) Sarazin et al. (2016); (10) Hindson et al (2014); (11) Akamatsu \& Kawahara (2013); (12) Akamatsu et al. (2013); (13) Thierbach et al. (2003); (14) Itahana et al. (2015); (15) van Weeren et al. (2016); (16) Kale et al. (2012).

one. This distance at $1.4 \mathrm{GHz}, 28 \mathrm{kpc}$, is smaller than the radio beam at same frequency $\left(69^{\prime \prime} \times 69^{\prime \prime}\right.$ or $\left.65 \times 65 \mathrm{kpc}\right)$ used for the spectral index estimation (Kale et al. 2012). Thus, $\mathcal{M}_{\mathrm{R}}$ may be affected by the ageing effect.

Additionally, the X-ray SB discontinuities in the west and east (see Figs. 13 and 14) do not coincide with the outermost edges of the radio relics. There is significant radio structure outside these SB discontinuities. Therefore, while the DSA mechanism associated to these shocks is assumed to be responsible for the radio emission directly behind the shocks, it cannot explain all radio emission. There might be other shocks further outward, but these are below the detection threshold using current instruments, due to a combination of low spatial resolution (Suzaku) or high background (XMM-Newton), so we cannot test that hypothesis in the present study. Together with the ageing effect discussed before, this might contribute to the difference between the Mach number derived from the X-ray and radio observations.

\subsection{Merger scenario}

The N-body hydrodynamical simulations of A3376 by Machado \& Lima Neto (2013) predict a merger scenario with two subclusters: one, eastern, which is more compact with four times more concentrated gas than the other, western subcluster. The eastern subcluster has a high initial velocity and is able to cross through the more massive western subcluster, disrupting its core and forming the dense and bright tail. This could be the reason why only one X-ray emission peak is found, probably associated to the BCG2 of the Eastern subcluster in the central region. This scenario is confirmed by the weak lensing analysis of MonteiroOliveira et al. (2017), which reveals that the mass peak concentration is in the stripped tail.

From the shock properties, we are able to derive the dynamical age of A3376. Assuming that the western and eastern shocks have traveled from the cluster core to the radio relic location with respective constant velocity $\left(v_{\text {shock, }} \sim 1630 \pm 220 \mathrm{~km} \mathrm{~s}^{-1}\right.$ and $v_{\text {shock,E }} \sim 1450 \pm 150 \mathrm{~km} \mathrm{~s}^{-1}$ ) and the distance between both shocks is $\sim 1.9 \mathrm{Mpc}$, the time required to reach the current position is $\sim 0.6$ Gyr. This value is in good agreement with the previous estimates by Monteiro-Oliveira et al. (2017), George et al. (2015) and Machado \& Lima Neto (2013). It might indicate that A3376 is a young merger cluster which is still evolving and following the outgoing scenario as proposed by Akamatsu et al. (2012) and Monteiro-Oliveira et al. (2017).

We also estimated the inclination angle of the line-of-sight with respect to the merging axis, assuming that the galaxies of the infalling (eastern) subcluster are moving together with the shock front. The brightest galaxy of the E subcluster has a $z=0.045591 \pm 0.00008$ (Smith et al. 2004) and the peculiar velocity with respect to the entire merging cluster $(z=0.0461 \pm 0.003$, Monteiro-Oliveira et al. 2017) is $\sim 154 \pm 94 \mathrm{~km} \mathrm{~s}^{-1}$. From the relation $\theta=\arctan \left(v_{\text {spec }} / v_{\text {shock,E }}\right)$, we estimated $\theta \sim 6^{\circ} \pm 4^{\circ}$. This means that the merger axis is close to the plane of sky.

\section{Cold front near the center}

The Chandra image shown in Fig. 17 reveals an arc-shape edge between $25-140^{\circ}$ (measured counter-clockwise), orthogonal to the merging axis in the NE direction. For a refined spatial analysis of this feature, we extracted the X-ray surface brightness profile from the center of A3376 to the E using the Chandra observations (see Table 1) in the $0.5-2.0 \mathrm{keV}$ band. The circular pie annuli used to accumulate the SB profile cover the angle interval $50-120^{\circ}$ from the centroid in $\mathrm{RA}=6^{\mathrm{h}} 02^{\mathrm{m}} 12^{\mathrm{s}} .08$, Dec $=-39^{\circ} 57^{\prime} 18^{\prime \prime} .53$ (same as the red annular sectors of Fig. 17). The same regions are used for the spectral analysis where point sources using the criteria of $S_{\mathrm{c}}=10^{-17} \mathrm{~W} \mathrm{~m} \mathrm{~m}^{-2}$ have been excluded. Figure 18 shows the radial SB profile along the E direction. It shows an edge $(C=1.78 \pm 0.15)$ in the SB profile at approximately $150 \mathrm{kpc}\left(\sim 3^{\prime}\right)$ distance from the X-ray emission peak.

We determined the radial temperature profile using XMM-Newton and Suzaku observations, see Fig. 19, across the E direction for the four annular regions shown in Fig. 17. The temperature rises from $\sim 3.0$ to $\sim 4.6 \mathrm{keV}\left(T_{\text {in }} / T_{\text {out }}=\right.$ 


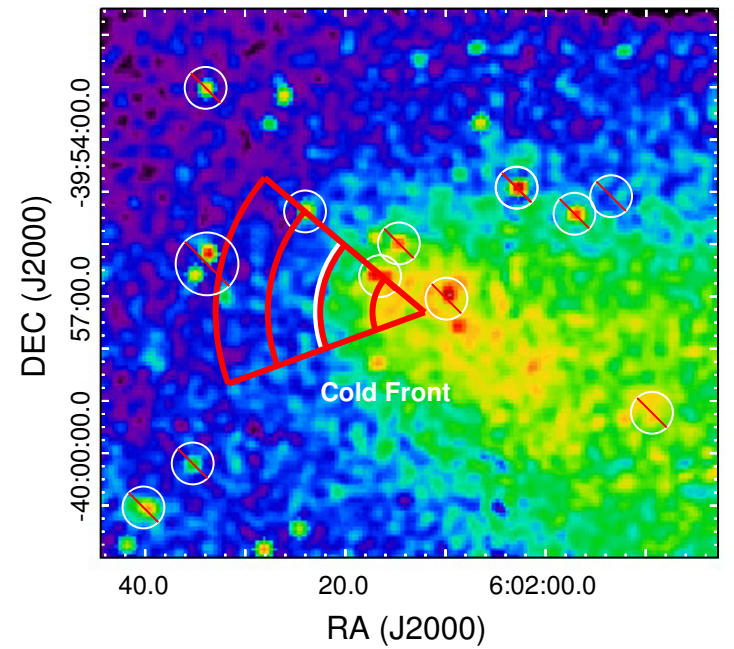

Fig. 17. A3376 Chandra background and exposure corrected image in the $0.5-2.0 \mathrm{keV}$ band. The red annular regions are used in the spectral analysis of the cold front and the same pie shaped annuli are used for the SB profile estimation.

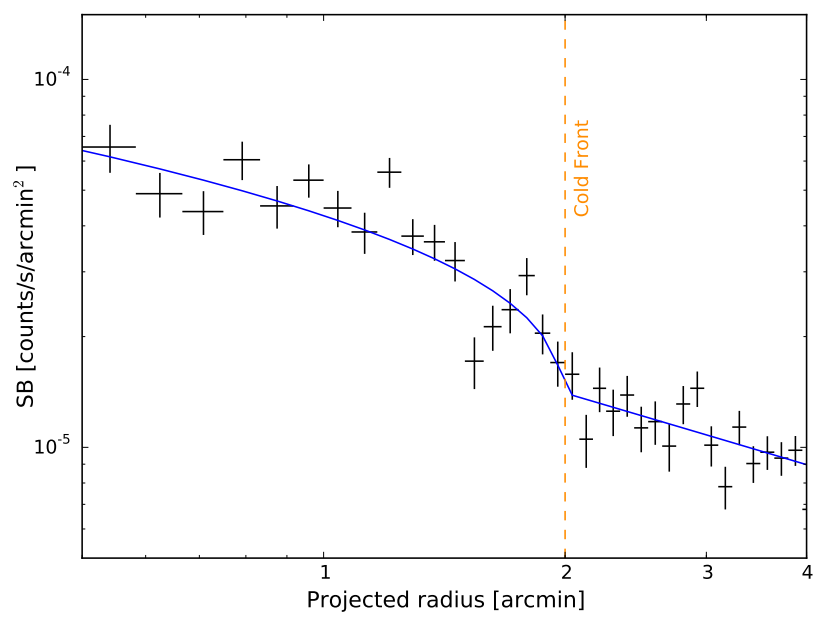

Fig. 18. Radial $X$-ray surface brightness profile across the cold front in the $0.5-2.0 \mathrm{keV}$ band using the Chandra observation. The profile is corrected for exposure and background, and point sources are removed. The solid blue curve is the best-fit model and the vertical orange line is the estimated position of the cold front.

$0.65 \pm 0.08)$ just outside of the SB edge location based on XMM-Newton observations.

The radial SB and temperature profiles show that the gas is cooler and denser (brighter) inside the edge. Using the density and temperature discontinuities of $C=1.78 \pm 0.15$ and $T_{\text {in }} / T_{\text {out }}=0.65 \pm 0.08$, respectively; the pressure jump is $P_{\text {in }} / P_{\text {out }}=1.16 \pm 0.17$. Therefore, the pressure is almost continuous across the edge, which agrees with the definition of a cold front.

In addition to the two shock fronts described in the previous sections, we found evidence of a sharp edge close to the center associated to a cold front. It delimits the boundaries of a cool and dense gas cloud moving through a hotter ambient gas, which is subject to ram pressure stripping (Markevitch \& Vikhlinin 2007; Vikhlinin et al. 2001). Figure 17 shows this case, with the shape of the front perpendicular to the merger axis advancing in the $\mathrm{NE}$ direction. Using the temperatures and densities derived from

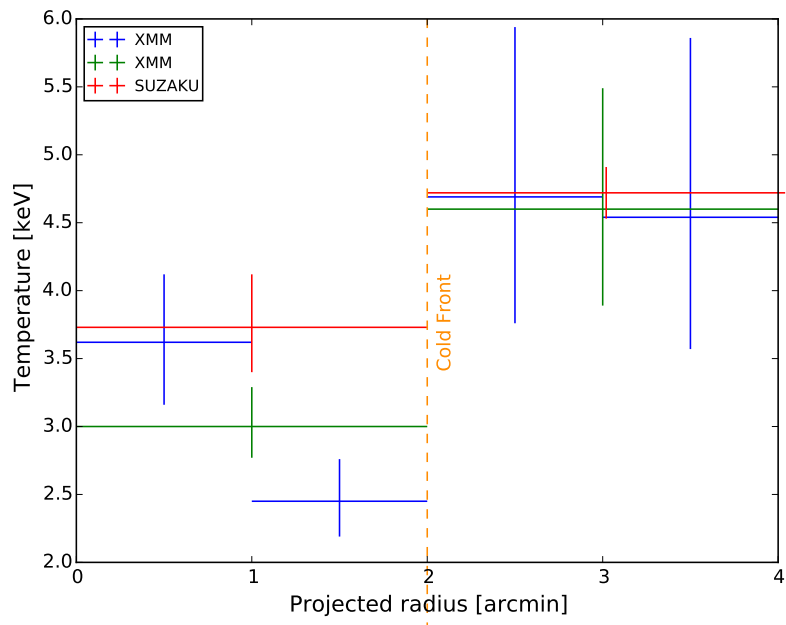

Fig. 19. Radial temperature distribution for the red annular regions shown in Fig. 17. Blue and green crosses correspond to XMM-Newton observations and red crosses to Suzaku. The vertical orange line shows the estimated position of the cold front.

the X-ray image and spectral analysis, we estimated the velocity of the cool gas cloud (Landau \& Lifshitz 1959; Vikhlinin et al. 2001; Dasadia et al. 2016b; Ichinohe et al. 2017). We approximated the cool gas as a spherical body advancing into ambient gas. If the cloud moves with supersonic velocity, a shock front could form upstream of the gas cloud. Near the cold front edge the cool gas decelerates to zero velocity at the stagnation point, referred here with the index 0 . We also assumed a free stream of gas upstream of the cold front, which is undisturbed and flows free, denoted by index 1 . The ratio of pressures in the free stream and the stagnation point can be expressed as a function of the gas cloud speed $v$ (Landau \& Lifshitz 1959):

$\frac{p_{0}}{p_{1}}= \begin{cases}\left(1+\frac{\gamma-1}{2} \mathcal{M}_{1}^{2}\right)^{\gamma /(\gamma-1)} & \left(\mathcal{M}_{1} \leq 1\right) \\ \left(\frac{\gamma+1}{2}\right)^{(\gamma+1) /(\gamma-1)} \mathcal{M}_{1}^{2}\left(\gamma-\frac{\gamma-1}{2 \mathcal{M}_{1}^{2}}\right)^{-1 /(\gamma-1)} & \left(\mathcal{M}_{1}>1\right)\end{cases}$

where $\gamma=5 / 3$ is the adiabatic index; $\mathcal{M}_{1}=v / c_{\mathrm{s}}$ and $c_{\mathrm{s}}$ are the Mach number and sound velocity of the free stream, respectively.

The gas parameter at the stagnation point cannot be measured directly, although the stagnation pressure is similar to the gas cloud inside the cold front (Vikhlinin et al. 2001). Therefore we assumed $T_{0}=3.00 \pm 0.27 \mathrm{keV}$ as the temperature inside the cold front and $T_{1}=4.60 \pm 0.80 \mathrm{keV}$ outside the cold front used as the free stream region (Vikhlinin et al. 2001; Sarazin et al. 2016). The density ratio of these regions is calculated from the best-fit parameter of the broken power-law model of Fig. $18, n_{0} / n_{1}=3.91 \pm 0.75$. Thus, the pressure ratio between the inside of the cold front and the outside free stream region is $p_{0} / p_{1}=2.6 \pm 0.6$, which corresponds to $\mathcal{M}_{1}=1.2 \pm 0.2$ in the free stream. Using $c_{\mathrm{cf}}=\sqrt{\gamma k T_{1} / \mu m_{p}}$ with $k T_{1}=4.60 \mathrm{keV}$, $c_{\mathrm{cf}}=1100 \pm 100 \mathrm{~km} \mathrm{~s}^{-1}$ and the velocity of the cool gas cloud is $v_{\mathrm{cf}}=1300 \pm 250 \mathrm{~km} \mathrm{~s}^{-1}$. This value is consistent with the velocity of the shock front, $v_{\text {shock, } \mathrm{E}}=1450 \pm 150 \mathrm{~km} \mathrm{~s}^{-1}$, derived in Sect. 4.3.

As explained in Dasadia et al. (2016b), for a rigid sphere the ratio of $d_{\mathrm{s}} / R_{c f}$, with $d_{\mathrm{s}}$ the shock "stand-off" distance and $R_{\mathrm{cf}}$ the radius of curvature of the cold front, depends on $\mathcal{M}$ as a function of $\left(\mathcal{M}^{2}-1\right)^{-1}$ (see Dasadia et al. Fig. 9, Schreier 1982; Vikhlinin et al. 2001; Sarazin 2001). For a shock with $\mathcal{M}_{E}=1.5 \pm 0.1$, 
Table 11. Temperature distribution at cold front regions shown in Fig. 18.

\begin{tabular}{ccc}
\hline \hline & Radius $\left(^{\prime}\right)$ & $k T(\mathrm{keV})$ \\
\hline & $0.5 \pm 0.5$ & $3.62 \pm 0.46$ \\
XMM-Newton & $1.5 \pm 0.5$ & $2.45 \pm 0.29$ \\
& $2.5 \pm 0.5$ & $4.69 \pm 1.14$ \\
& $3.5 \pm 0.5$ & $4.54 \pm 1.15$ \\
\hline XMM-Newton & $1.0 \pm 1.0$ & $3.00 \pm 0.27$ \\
& $3.0 \pm 1.0$ & $4.60 \pm 0.80$ \\
\hline Suzaku & $1.0 \pm 1.0$ & $3.73 \pm 0.36$ \\
& $3.0 \pm 1.0$ & $4.71 \pm 0.19$ \\
\hline
\end{tabular}

the shock offset ratio is $d_{\mathrm{s}} / R_{\mathrm{cf}} \sim 0.8 \pm 0.1, d_{\mathrm{s}} \sim 120 \pm 15 \mathrm{kpc}$ $\left(R_{\mathrm{cf}} \sim 150 \mathrm{kpc}\right)$. This separation for a rigid sphere is smaller than the distance of $\sim 300 \mathrm{kpc}$ between the cold front and shock front present in the east. This behavior is the same for most of the observed clusters (Dasadia et al. 2016b). As explained by Sarazin et al. (2016), after the first core passage the shock accelerates toward the periphery of the clusters, while the cool gas is decelerated by gravity and ram pressure. For this reason, the shock is expected to move away from the cold front and the separation between both features might be larger than the rigid sphere model predicts.

\section{Summary}

We present a spectral analysis of A3376 in four directions (west, east, north, and south) using Suzaku deep observations and supported by XMM-Newton and Chandra observations. A3376 is a merging galaxy cluster with two shock fronts and one cold front confirmed. One shock is coincident with the radio relic in the west and the other might be associated to the "notch" of the eastern relic. Shocks are moving with a Mach number $\mathcal{M} \sim 2-3$. The cold front is located at approximately $150 \mathrm{kpc}\left(r \sim 3^{\prime}\right)$ from the $\mathrm{X}$-ray emission peak at the center and delimits a cool gas cloud moving with $v \sim 1300 \mathrm{~km} \mathrm{~s}^{-1}$.

We determine the ICM structure up to $0.9 r_{200}$ in the W, $0.6 r_{200}$ in the E. We observe a temperature enhancement followed by a drop at $\sim 0.7 r_{200}$ for the $\mathrm{W}$ and $\sim 0.5 r_{200}$ for the $\mathrm{E}$. We confirm temperature and surface brightness discontinuities in both directions. In the west it coincides with the radio relic position and in the east, it is located at $450 \mathrm{kpc}\left(r \sim 8^{\prime}\right)$. The temperature structure in the south follows the simulated profile of Burns et al. (2010) for relaxed clusters.

We estimate the Mach number based on the temperature jump, being $\mathcal{M}_{\mathrm{W}}=2.8 \pm 0.4$ and $\mathcal{M}_{\mathrm{E}}=1.5 \pm 0.1$, for the western and eastern region, respectively. We derive the shock speed velocity as $v_{\text {shock, } \mathrm{W}} \sim 1630 \pm 220 \mathrm{~km} \mathrm{~s}^{-1}$ and $v_{\text {shock,E }} \sim 1450 \pm 150 \mathrm{~km} \mathrm{~s}^{-1}$.

Assuming that the shock fronts are moving with constant velocity, the time since core passage is $\sim 0.6 \mathrm{Gyr}$, which is in good agreement with the N-body hydrodynamical simulations of Machado \& Lima Neto (2013), and with earlier multiwavelength analyses of George et al. (2015) and Monteiro-Oliveira et al. (2017). Combining the eastern shock velocity and the peculiar velocity of the eastern brightest galaxy let us infer that the merger axis is close to the plane of the sky.
We compare the Mach numbers inferred from the temperature discontinuities with those derived from radio observations assuming the DSA mechanism. Not all the clusters present a consistent correlation between X-ray and radio Mach numbers. For A3376 the Mach number for the western relic estimated by Kale et al. (2012) is consistent with our result.

Acknowledgements. The authors thank the anonymous referee for constructive comments and suggestions. The authors also thank Dr. R. Kale for providing the VLA radio data. I.U. thanks H. Martínez-Rodríguez for his support in the initial stage of this work. H.A. acknowledges the support of NWO via Veni grant. T.O. and Y.I. acknowledge support by JSPS KAKENHI Grant Numbers 26220703 and $15 \mathrm{H} 03642$. SRON is supported financially by NWO, the Netherlands Organization for Scientific Research. This work is based on observations obtained with XMM-Newton, an ESA science mission with instruments and contributions directly funded by ESA member states and the USA (NASA).

\section{References}

Akamatsu, H., \& Kawahara, H. 2013, PASJ, 65, 16

Akamatsu, H., Takizawa, M., Nakazawa, K., et al. 2012, PASJ, 64

Akamatsu, H., Inoue, S., Sato, T., et al. 2013, PASJ, 65, 89

Akamatsu, H., van Weeren, R. J., Ogrean, G. A., et al. 2015, A\&A, 582, A20

Akamatsu, H., Mizuno, M., Ota, N., et al. 2017, A\&A, 600, A100

Bagchi, J., Durret, F., Lima Neto, G. B., \& Paul, S. 2006, Science, 314, 791

Bell, A. R. 1987, MNRAS, 225, 615

Blandford, R., \& Eichler, D. 1987, Phys. Rep., 154, 1

Blanton, E. L., Sarazin, C. L., \& McNamara, B. R. 2003, ApJ, 585, 227

Bonafede, A., Intena, H. T., Brüggen, M., et al. 2014, ApJ, 785, 1

Botteon, A., Gastaldello, F., Brunetti, G., \& Dallacasa, D. 2016a, MNRAS, 460, 84

Botteon, A., Gastaldello, F., Brunetti, G., \& Kale, R. 2016b, MNRAS, 463, 1534 Bourdin, H., Mazzotta, P., Markevitch, M., Giacintucci, S., \& Brunetti, G. 2013, ApJ, 764, 82

Brüggen, M., Bykov, A., Ryu, D., \& Röttgering, H. 2012, Space Sci. Rev., 166, 187

Brunetti, G., \& Jones, T. W. 2014, IJMPD, 23, 1430007

Burns, J. O., Skillman, S. W., \& O'Shea, B. W. 2010, ApJ, 721, 1105

Cappelluti, N., Li, Y., Ricarte, A., et al. 2017, ApJ, 837, 19

Cash, W. 1979, ApJ, 228, 939

Dasadia, S., Sun, M., Sarazin, C., et al. 2016a, ApJ, 820, L20

Dasadia, S., Sun, M., Morandi, A., et al. 2016b, MNRAS, 458, 681

De Grandi, S., \& Molendi, S. 2002, ApJ, 567, 163

Drury, L. O. 1983, Rep. Prog. Phys., 46, 973

Durret, F., Perrot, C. Lima Neto, G. B., et al. 2013, A\&A, 560, A78

Eckert, D., Ettori, S., Coupon, J., et al. 2016a, A\&A, 592, A12

Eckert, D., Jauzac, M., Vazza, F., et al. 2016b, MNRAS, 461, 1302

Eckert, D., Molendi, S., \& Paltani, S. 2011, A\&A, 526, A79

Feretti, L., Giovannini, G., Govoni, F., \& Murgia, M. 2012, A\&ARv, 20, 54

Ferrari, C., Govoni, F., Schindler, S., Bykov, A. M., \& Rephaeli, Y. 2008, Space Sci. Rev., 134, 93

Finoguenov, A., Sarazin, C. L., Nakazawa, K., Wik, D. R., \& Clarke, T. E. 2010, ApJ, 715, 1143

Fujita, Y., Akamatsu, H., \& Kimura, S. S. 2016, PASJ, 68, 34

Fujita, Y., Tawa, N., Hayashida, K., et al. 2008, PASJ, 60, 343

Fujita, Y., Takizawa, M., Yamazaki, R., Akamatsu, H., \& Ohno, H. 2015, ApJ, 815,116

George, L. T., Dwarakanath, K. S., Johnston-Hollitt, M., et al. 2015, MNRAS, 451, 4207

Giacintucci, S., Venturi, T., Macario, G., et al. 2008, A\&A, 486, 347

Guo, X., Sironi, L., \& Narayan, R. 2014a, ApJ, 794, 153

Guo, X., Sironi, L., \& Narayan, R. 2014b, ApJ, 797, 47

Guo, X., Sironi, L., \& Narayan, R. 2017, ApJ, 851, 134

Henry, J. P., Evrard, A. E., Hoekstra, H., Babul, A., \& Mahdavi, A. 2009, ApJ, 691, 1307

Hindson, L., Johnston-Hollitt, M., Hurley-Walker, N., et al. 2014, MNRAS, 445, 330

Hoang, D. N., Shimwell, T. W., Stroe, A., et al. 2017, MNRAS, 471, 1107

Hoshino, A., Henry, J. P., Sato, K., et al. 2010, PASJ, 62, 371

Ichinohe, Y., Simionescu, A., Werner, N., \& Takahashi, T. 2017, MNRAS, 467, 3662

Ishisaki, Y., Maeda, Y., Fujimoto, R., et al. 2007, PASJ, 59, 113

Itahana, M., Takizawa, M., Akamatsu, H., et al. 2015, PASJ, 67, 113

Kaastra, J. S. 2017, A\&A, 605, A51

Kaastra, J. S., \& Bleeker, J. A. M. 2016, A\&A, 587, A151 
Kaastra, J. S., Mewe, R., \& Nieuwenhuijzen, H. 1996, in UV and X-ray Spectroscopy of Astrophysical and Laboratory Plasmas, eds. K. Yamashita, \& T. Watanabe, 411-414

Kale, R., Dwarakanath, K. S., Bagchi, J., \& Paul, S. 2012, MNRAS, 426, 1204 Kang, H. 2017, JKAS, 50, 93

Kang, H., \& Ryu, D. 2015, ApJ, 809, 186

Kang, H., \& Ryu, D. 2016, ApJ, 823, 13

Kang, H., Ryu, D., \& Jones, T. W. 2012, ApJ, 756, 97

Kawano, N., Fukazawa, Y., Nishino, S., et al. 2009, PASJ, 61, 377

Koyama, K., Tsunemi, H., Dotani, T., et al. 2007, PASJ, 59, S23

Kushino, A., Ishisaki, Y., Morita, U., et al. 2002, PASJ, 54, 327

Landau, L. D., \& Lifshitz, E. M. 1959, Fluid mechanics, Course of theoretical physics (Oxford: Pergamon Press)

Lindner, R. R., Baker, A. J., Hughes, J. P., et al. 2014, ApJ, 786, 49

Lodders, K., Palme, H., \& Gail, H.-P. 2009, in Landolt Bömstein (Heidelberg: Springer-Verlag Berlin), 4B, 712

Macario, G., Markevitch, M., Giacintucci, S., et al. 2011, ApJ, 782, 82

Machado, R. E. G., \& Lima Neto, G. B. 2013, MNRAS, 430, 3249

Markevitch, M., \& Vikhlinin, A. 2007, Phys. Rep., 443, 1

Markevitch, M., Gonzalez, A. H., David, L., et al. 2002, ApJ, 567, L27

Markevitch, M., Govoni, F., Brunetti, G., \& Jerius, D. 2005, ApJ, 627, 733

Mazzota, P., Bourdin, H., Giacintucci, S., Markevitch, M., \& Venturi, T. 2011, Mem. Soc. Astron. It., 82, 495

Miniati, F. 2002, MNRAS, 337, 199

Mitsuda, K., Bautz, M., Inoue, H., et al. 2007, PASJ, 59, 1

Monteiro-Oliveira, R., Neto, G. B. L., Cypriano, E. S., et al. 2017, MNRAS, 468, 4566

Ogrean, G. A., \& Brüggen, M. 2013, MNRAS, 433, 1701

Ogrean, G. A., Brüggen, M., van Weeren, R. J., Burgmeier, A., \& Simionescu, A. 2014, MNRAS, 443, 2463

Owers, M. S., Nulsen, P. E. J., Couch, W. J., et al. 2014, ApJ, 780, 163

Pacholczyk, A. G. 1973, Radio astrophysics. Nonthermal processes in galactic and extragalactic sources (Moskva: Mir)

Paul, S., Iapichino, L., Miniati, F., Bagchi, J., \& Mannheim, K. 2011, ApJ, 726 17

Pinzke, A., Oh, S. P., \& Pfrommer, C. 2013, MNRAS, 435, 1061
Pizzo, R. F., \& de Bruyn, A. G. 2009, A\&A, 507, 639

Reiprich, T. H., Hudson, D. S., Zhang, Y. Y., et al. 2009, A\&A, 501, 899

Reiprich, T. H., Basu, K., Ettori, S., et al. 2013, Space Sci. Rev., 177, 195

Russell, H. R., Mcnamara, B. R., Sanders, J. S., et al. 2012, MNRAS, 423, 236

Russell, H. R., Fabian, A. C., McNamara, B. R., et al. 2014, MNRAS, 444, 629

Sarazin, C. L. 2001, Merging Processes in Clusters of Galaxies, Astrophysics and Space Science Library (Dordrecht: Kluwer Academic Publishers), 272, 1

Sarazin, C. L., Finoguenov, A., Wik, D. R., \& Clarke, T. E. 2016, ApJ, submitted, [arXiv:1606.07433]

Schreier, S. 1982, in Compressible Flow. Wiley, New York

Serlemitsos, P. J., Soong, Y., Chan, K. W., et al. 2007, PASJ, 59, 9

Shimwell, T. W., Markevitch, M., Brown, S., et al. 2015, MNRAS, 449, 1486

Shimwell, T. W., Luckin, J., Br, M., et al. 2016, MNRAS, 459, 277

Skillman, S. W., Xu, H., Hallman, E. J., et al. 2013, ApJ, 765

Smith, R. J., Hudson, M. J., Nelan, J. E., et al. 2004, ApJ, 128, 1558

Storm, E., Vink, J., Zandanel, F., \& Akamatsu, H. 2018, MNRAS, 479, 553

Stroe, A., Harwood, J. J., Hardcastle, M. J., \& Röttgering, H. J. 2014, MNRAS, 445,1213

Struble, M. F., \& Rood, H. J. 1999, ApJS, 125, 35

Tawa, N., Hayashida, K., Nagai, M., et al. 2008, PASJ, 60, 11

Thierbach, M., Klein, U., \& Wielebinski, R. 2003, A\&A, 397, 53

Trasatti, M., Akamatsu, H., Lovisari, L., et al. 2015, A\&A, 575, A45

Urban, O., Werner, N., Allen, S. W., Simionescu, A., \& Mantz, A. 2017, MNRAS, 470, 4583

van Weeren, R. J., Röttgering, H. J. A., Rafferty, D. A., et al. 2012, A\&A, 543, A43

van Weeren, R. J., Andrade-Santos, F., Dawson, W. A., et al. 2017, Nat. Astron., 1,5

van Weeren, R. J., Brunetti, G., Brüggen, M., et al. 2016, ApJ, 818, 204

Vazza, F., \& Brüggen, M. 2014, MNRAS, 437, 2291

Vikhlinin, A., Markevitch, M., \& Murray, S. S. 2001, ApJ, 551, 160

Vink, J., \& Yamazaki, R. 2014, ApJ, 780, 125

Willingale, R., Starling, R. L. C., Beardmore, A. P., Tanvir, N. R., \& O'Brien, P. T. 2013, MNRAS, 431, 394 